\title{
ISG15-dependent activation of the sensor MDA5 is antagonized by the SARS-CoV-2 papain-like protease to evade host innate immunity
}

\author{
GuanQun Liu $\mathbb{1}^{1,2,4}$, Jung-Hyun Lee $\mathbb{1}^{1,2,4}$, Zachary M. Parker ${ }^{2}$, Dhiraj Acharya ${ }^{1,2}$, Jessica J. Chiang ${ }^{3}$, \\ Michiel van Gent ${ }^{1,2}$, William Riedl1,2, Meredith E. Davis-Gardner ${ }^{3}$, Effi Wies ${ }^{3}$, Cindy Chiang ${ }^{1,2}$ and \\ Michaela U. Gack $\mathbb{1}^{1,2} \bowtie$
}

\begin{abstract}
Activation of the RIG-I-like receptors, retinoic-acid inducible gene I (RIG-I) and melanoma differentiation-associated protein 5 (MDA5), establishes an antiviral state by upregulating interferon (IFN)-stimulated genes (ISGs). Among these is ISG15, the mechanistic roles of which in innate immunity still remain enigmatic. In the present study, we report that ISG15 conjugation is essential for antiviral IFN responses mediated by the viral RNA sensor MDA5. ISGylation of the caspase activation and recruitment domains of MDA5 promotes its oligomerization and thereby triggers activation of innate immunity against a range of viruses, including coronaviruses, flaviviruses and picornaviruses. The ISG15-dependent activation of MDA5 is antagonized through direct de-ISGylation mediated by the papain-like protease of SARS-CoV-2, a recently emerged coronavirus that has caused the COVID-19 pandemic. Our work demonstrates a crucial role for ISG15 in the MDA5-mediated antiviral response, and also identifies a key immune evasion mechanism of SARS-CoV-2, which may be targeted for the development of new antivirals and vaccines to combat COVID-19.
\end{abstract}

V iral perturbation of host immune homoeostasis is monitored by the innate immune system, which relies on receptors that sense danger- or pathogen-associated molecular patterns (PAMPs) ${ }^{1-3}$. The RIG-I-like receptors (RLRs) RIG-I and MDA5 are pivotal for virus detection by surveying the cytoplasm for viral or host-derived immunostimulatory RNAs ${ }^{4}$. Binding of RNA to the C-terminal domain (CTD) and helicase of RIG-I and MDA5 leads to their signalling-primed conformation that allows for the recruitment of several enzymes ${ }^{5}$. These enzymes modify RLRs at multiple domains and sites, and post-translational modifications (PTMs) are particularly well studied for the caspase activation and recruitment domains (CARDs), the signalling modules. Protein phosphatase 1 (PP1) $\alpha / \gamma$ dephosphorylates the RIG-I and MDA5 CARDs ${ }^{6}$. In the case of RIG-I, dephosphorylation promotes Lys63-linked polyubiquitination of the CARDs by TRIM25 (tripartite motif containing 25) and other E3 ligases ${ }^{7,8}$, which stabilizes the oligomeric form of RIG-I, thereby enabling mitochondrial antiviral-signalling protein (MAVS) binding. Compared with those of RIG-I, the individual steps of MDA5 activation and critical PTMs involved are less well understood.

RLR activation induces the production of type I and III IFNs which, in turn, propagate antiviral signalling by upregulating ISGs $^{9,10}$. Among those is ISG15, a ubiquitin-like protein that can be covalently conjugated to lysine residues of target proteins, a PTM process termed ISGylation ${ }^{11}$. Although ISG15 conjugation has been widely recognized to act antivirally ${ }^{12}$, mechanisms of host protein ISGylation that could explain the broad antiviral restriction activity of ISG15 are currently unknown.

The causative agent of the ongoing COVID-19 pandemic, severe acute respiratory syndrome coronavirus 2 (SCoV2), belongs to the
Coronaviridae family that contains several other human pathogens. Coronaviruses have an exceptional capability to suppress IFN-mediated antiviral responses, and low IFN production in SCoV2-infected patients correlated with severe disease ${ }^{13}$. Among the coronaviral IFN antagonists is the papain-like protease (PLpro) which has deubiquitinating and de-ISGylating activities ${ }^{14,15}$.

In the present study, we identify an essential role for ISGylation in MDA5 activation. We further show that SCoV2 PLpro interacts with MDA5 and antagonizes ISG15-dependent MDA5 activation via active de-ISGylation, unveiling that SCoV2 has already evolved to escape immune surveillance by MDA5.

\section{Results}

MDA5, but not RIG-I, signalling requires ISG15. To identify PTMs of the MDA5 CARDs that may regulate MDA5 activation, we subjected affinity-purified MDA5-2CARD fused to glutathione-S-transferase (GST-MDA5-2CARD), or GST alone, to liquid chromatography coupled with tandem mass spectrometry (LC-MS/MS), and found that, specifically, GST-MDA5-2CARD co-purified with ISG15, which appeared as two bands that migrated more slowly (by $\sim 15$ and $30 \mathrm{kDa}$ ) than unmodified GST-MDA52CARD (ExtendedData Fig. 1a).Immunoblotting(IB)confirmed that GST-MDA5-2CARD is modified by ISG15 (Extended Data Fig. 1b). We next determined the relevance of ISG15 for MDA5-induced signalling. Whereas FLAG-MDA5 expression in wild-type (WT) mouse embryonic fibroblasts (MEFs) induced IFN- $\beta$ messenger RNA and protein as well as $\mathrm{Ccl} 5$ transcripts in a dose-dependent manner, FLAG-MDA5 expression in Isg15 $5^{-/}$MEFs led to ablated antiviral gene and protein expression (Fig. 1a and Extended Data Fig. 1c). Similarly, antiviral gene induction by FLAG-MDA5 was

'Florida Research and Innovation Center, Cleveland Clinic, Port St Lucie, FL, USA. '2Department of Microbiology, University of Chicago, Chicago, IL, USA. ${ }^{3}$ Department of Microbiology and Immunobiology, Harvard Medical School, Boston, MA, USA. ${ }^{4}$ These authors contributed equally: GuanQun Liu, Jung-Hyun Lee. 巴e-mail: gackm@ccf.org 

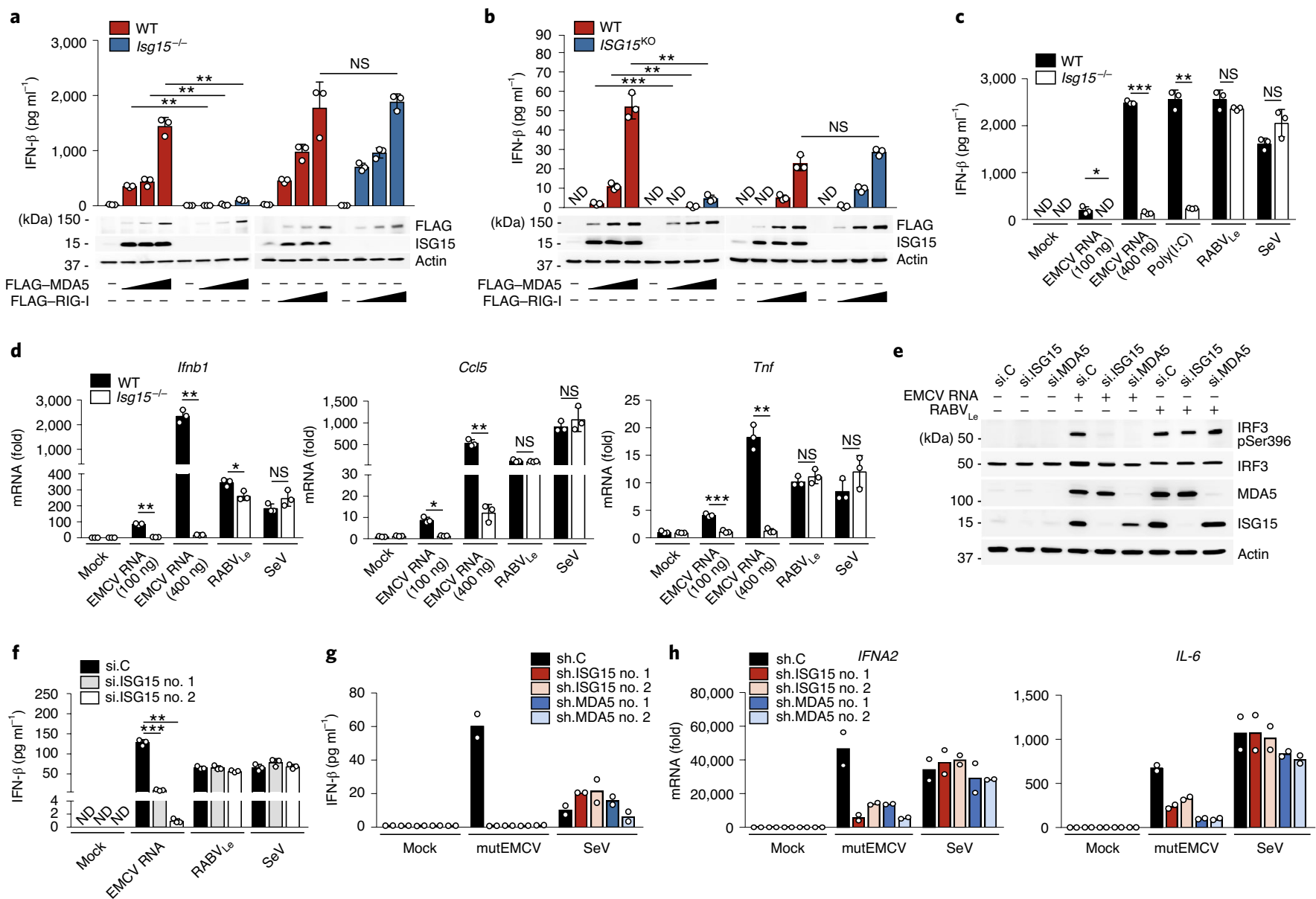

Fig. 1 | ISGylation is required for MDA5, but not RIG-I, signalling. a,b, ELISA of IFN- $\beta$ from supernatants of MEFs (WT or Isg15 $5^{-/-}$) (a) and HeLa cells (WT or ISG15 KO) (b) transiently transfected with increasing amounts of FLAG-tagged MDA5 or RIG-I for $40 \mathrm{~h}$. Whole-cell lysates (WCLs) were probed by IB with anti-ISG15, anti-FLAG and anti-actin (loading control). c, ELISA of IFN- $\beta$ from supernatants of WT or Isg15-1- MEFs that were mock stimulated

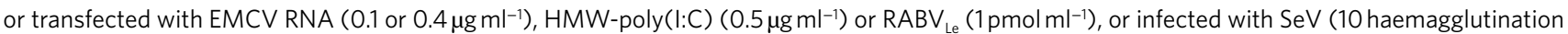
units (HAU) $\mathrm{ml}^{-1}$ ) for $24 \mathrm{~h}$. d, RT-qPCR analysis of Ifnb1, CcI5 and Tnf mRNA in WT and Isg15-/- MEFs stimulated as in c. e, IRF3 phosphorylation in the WCLs of NHLFs that were transfected with the indicated siRNAs for $30 \mathrm{~h}$ and then mock stimulated or transfected with EMCV RNA $\left(0.4 \mu \mathrm{g} \mathrm{ml}^{-1}\right)$ or

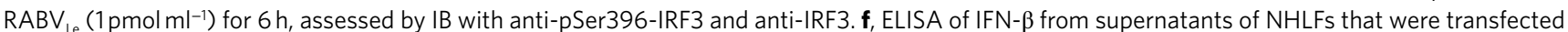
with the indicated siRNAs for $30 \mathrm{~h}$ and then mock stimulated or transfected with EMCV RNA $\left(0.4 \mu \mathrm{g} \mathrm{ml}^{-1}\right)$ or RABV $\left(1 \mathrm{pmol} \mathrm{ml}^{-1}\right)$, or infected with SeV $\left(10 \mathrm{HAU} \mathrm{ml}^{-1}\right)$ for $16 \mathrm{~h} . \mathbf{g}$, ELISA of IFN- $\beta$ from the supernatants of PBMCs that were transduced for $40 \mathrm{~h}$ with the indicated shRNA lentiviral particles and then infected with mutEMCV $(\mathrm{MOI}=10)$ or $\mathrm{SeV}\left(200 \mathrm{HAU} \mathrm{ml}^{-1}\right)$ for $8 \mathrm{~h} . \mathbf{h}, \mathrm{RT}$-qPCR analysis of IFNA2 and IL-6 mRNA in PBMCs that were transduced and infected as in $\mathbf{g}$. Data represent at least two independent experiments with similar results (mean \pm s.d. of $n=3$ biological replicates in a-d and $\mathbf{f}$, and mean of $n=2$ biological replicates in $\mathbf{g}$ and $\mathbf{h}$ ). ${ }^{\star} P<0.05,{ }^{\star \star} P<0.01,{ }^{\star \star \star} P<0.001$ (two-tailed, unpaired Student's t-test). ND, not detected; NS, not significant.

strongly diminished in ISG15 knockout (KO) HeLa (human) cells compared with WT control cells (Fig. 1b and Extended Data Fig. 1d), ruling out a species-specific effect. In contrast, FLAG-RIG-I induced comparable amounts of secreted IFN- $\beta$ protein as well as Ifnb1 and Ccl5 transcripts in Isg15 ${ }^{-1-}$ and WT MEFs (Fig. 1a and Extended Data Fig. 1c). IFNB1 and CCL5 transcripts as well as IFN- $\beta$ protein production by FLAG-RIG-I were similar or slightly enhanced in ISG15 KO HeLa cells compared with WT cells (Fig. 1b and Extended Data Fig. 1d), consistent with previous reports that ISGylation negatively impacts RIG-I signalling ${ }^{16,17}$.

We next tested the effect of ISG15 gene deletion on the activation of endogenous MDA5 and RIG-I by their respective ligands. IFN- $\beta$ production as well as IFNB1, CCL5 and TNF gene expression induced by transfection of encephalomyocarditis virus (EMCV) RNA or high-molecular-weight (HMW)-poly(I:C), both of which are predominantly sensed by MDA5, were profoundly attenuated in Isg15 ${ }^{-1-}$ MEF, ISG15 KO HeLa and ISG15 KO HAP-1 cells compared with their respective control cells (Fig. 1c,d and Extended Data Fig. 1e-g). Importantly, the ablation of antiviral gene induction by EMCV RNA or HMW-poly(I:C) in ISG15 KO cells was not due to abrogated MDA5 gene expression; on the contrary, MDA5 mRNA expression was enhanced in ISG15 KO cells compared with WT cells (Extended Data Fig. 1f,g). In contrast to stimulation with MDA5 agonists, stimulation of Isg15 ${ }^{-/-}$MEFs and ISG15 KO HeLa cells by rabies virus leader RNA $\left(\mathrm{RABV}_{\mathrm{Le}}\right)$ transfection or Sendai virus $(\mathrm{SeV})$ infection, which are RIG-I stimuli, led to IFN- $\beta$ production and antiviral gene expression comparable to WT cells (Fig. 1c,d and Extended Data Fig. 1e). To rule out potential clonal effects that could be associated with ISG15 gene-deleted cells, we performed transient gene-silencing experiments in primary normal human lung fibroblasts (NHLFs). ISG15 silencing, similar to MDA5 knockdown, led to an almost-complete loss of phosphorylation of IFN-regulatory factor 3 (IRF3) - a hallmark of RLR-signal activation-following stimulation with EMCV RNA but not $\mathrm{RABV}_{\mathrm{Le}}$ 
(Fig. 1e). In agreement with this, ISG15 knockdown greatly diminished IFN- $\beta$ production as well as antiviral transcript expression in NHLFs transfected with EMCV RNA, but not in cells stimulated with $\mathrm{RABV}_{\mathrm{Le}}$ or SeV (Fig. If and Extended Data Fig. 1h). Small hairpin (sh)RNA-mediated silencing of ISG15 or MDA5 in primary human peripheral blood mononuclear cells (PBMCs) also substantially reduced antiviral protein and transcript expression after infection with a recombinant mutant EMCV (mutEMCV) deficient in MDA5 antagonism ${ }^{18,19}$, compared with infected PBMCs transduced with non-targeting control shRNA (Fig. 1g,h and Extended Data Fig. 1i). By contrast, ISG15 or MDA5 depletion did not affect cytokine responses in PBMCs after $\mathrm{SeV}$ infection (Fig. $1 \mathrm{~g}$, h and Extended Data Fig. 1i). These results show that ISG15 is essential for immune signalling by MDA5, but not RIG-I.

The MDA5 CARDs are ISGylated at Lys23 and Lys43. To corroborate our MS analysis that identified MDA5-2CARD ISGylation, we first tested whether endogenous MDA5 is also modified by ISG15. Endogenous MDA5 was robustly ISGylated in cells transfected with HMW-poly(I:C), or infected with dengue (DENV) or Zika (ZIKV) viruses that are sensed by MDA5 (ref. ${ }^{5}$ ) (Fig. 2a). Notably, endogenous MDA5 was also ISGylated in uninfected cells, although at very low levels (Extended Data Fig. 2a), which is consistent with previous findings that many host proteins are also ISGylated at low levels in normal (uninfected) conditions ${ }^{20}$. In cells treated with anti-IFNAR2 to block IFNAR-signalling-mediated ISG upregulation, ISG15 or MDA5 silencing led to a comparable reduction of IFNB1 gene expression after mutEMCV infection (Extended Data Fig. 2b), indicating that ISG15-dependent MDA5 signalling occurs even in the absence of IFNAR signalling.

Biochemical analysis confirmed that the MDA5-2CARD, but not MDA5 2 CARD (containing helicase and CTD), is the primary site of MDA5 ISGylation showing two prominent bands for ISGylated 2CARD (Fig. 2b). Reconstitution of ISG15 KO HeLa cells with either WT ISG15, or an unconjugatable ISG15 mutant in which the two glycines needed for conjugation were replaced with alanine (ISG15-AA) ${ }^{21}$, demonstrated covalent ISG15 conjugation (Fig. 2c).

Mutation of individual lysine residues in GST-MDA5-2CARD to arginine revealed that single-site mutation of Lys23 and Lys43 noticeably reduced ISGylation (Extended Data Fig. 2c), whereas their combined mutation (Lys23Arg/Lys43Arg) almost abolished ISGylation (Fig. 2d). Full-length FLAG-MDA5 Lys23Arg/Lys43Arg also showed markedly diminished ISGylation (Fig. 2e and Extended Data Fig. 2d); the residual ISGylation seen in FLAG-MDA5 Lys23Arg/Lys43Arg is probably due to additional minor sites in the 2CARD and/or $\triangle 2$ CARD. Of note, the Lys23Arg/Lys43Arg mutation had no effect on MDA5-2CARD SUMOylation ${ }^{5}$ (Extended Data Fig. 2e). Furthermore, whereas RIG-I-2CARD was robustly ubiquitinated (which represents covalent Lys63-linked ubiquitination $^{7}$ ), neither MDA5-2CARD WT nor the Lys23Arg/Lys43Arg mutant showed detectable levels of ubiquitination (Extended Data Fig. 2f). Collectively, these results indicate that the MDA5 CARDs undergo ISGylation at two major sites, Lys23 and Lys43.

CARD ISGylation is required for MDA5 activation. When comparing their signal-transducing ability, MDA5-2CARD Lys23Arg and Lys43Arg single-site mutants showed partially reduced IFN- $\beta$ promoter activation compared with WT MDA5-2CARD, whereas the Lys23Arg/Lys43Arg mutant had a profoundly reduced signalling activity, which was almost as strong as that of the signalling-defective mutants Ser88Glu and Ser88Asp ${ }^{6}$ (Extended Data Fig. 2g). In contrast, a mutant in which Lys68, which is the lysine residue that is most proximal to Lys43 and Lys23, was substituted with arginine (Lys68Arg) showed comparable ISG15 conjugation and signalling competency to WT 2CARD (Extended Data Fig. 2c,g). MDA5-2CARD Lys23Arg/Lys43Arg, in contrast to WT MDA5-2CARD, also failed to induce IRF3 dimerization (Extended Data Fig. 2h). FLAG-MDA5 Lys23Arg, Lys43Arg or Lys23Arg/Lys43Arg also showed reduced, or almost abolished, IFN- $\beta$ promoter-activating abilities, compared with FLAG-MDA5 WT (Fig. 2f). MDA5 Lys23Arg/Lys43Arg showed a profound signalling defect even when expressed at high amounts, whereas WT MDA5 induced antiviral transcripts in a dose-dependent manner (Fig. 2g). In agreement, STAT1 phosphorylation, a hallmark of IFNAR signalling, as well as ISG protein expression were highly induced by MDA5 WT, but not Lys23Arg/Lys43Arg (Fig. 2h). Complementation of MDA5-gene-edited human astrocytes (SVGAs) with MDA5 Lys23Arg/Lys43Arg or Ser88Glu led to greatly diminished IFNB1, CCL5 and ISG transcripts compared with cells expressing WT MDA5 (Fig. 2i and Extended Data Fig. 2i). These results demonstrate that ISGylation at Lys 23 and Lys43 is essential for MDA5-mediated cytokine responses.

Dephosphorylation by PP1 regulates MDA5 ISGylation. Similar to RIG-I, MDA5 is phosphorylated within the CARDs in uninfected cells, which prevents autoactivation; dephosphorylation of RIG-I and MDA5 by PP $1 \alpha / \gamma$ is crucial for unleashing RLRs from their signalling-repressed states ${ }^{6,22-24}$. Dephosphorylation of RIG-I allows Lys63-linked ubiquitination of the CARDs, which promotes RIG-I multimerization and signalling 5 . The details of how CARD dephosphorylation (at Ser88) triggers MDA5 activation have remained elusive, and therefore we tested whether dephosphorylation regulates MDA5 ISGylation. Silencing of PP1 $\alpha / \gamma$ strongly diminished MDA5-2CARD ISGylation (Extended Data Fig. 3a). Furthermore, the phosphomimetic Ser88Glu and Ser88Asp mutants had reduced ISGylation, whereasthephospho-nullSer88Ala mutantshowed stronger ISGylation than WT MDA5-2CARD (Extended Data Fig. 3b). Conversely, MDA5 WT and Lys23Arg/Lys43Arg had comparable Ser88 phosphorylation (Extended Data Fig. 3c). Together, these data suggest that MDA5 dephosphorylation at Ser88 precedes CARD ISGylation.

We next made use of the measles virus $\mathrm{V}$ protein $(\mathrm{MeV}-\mathrm{V})$, which antagonizes MDA5 Ser88 dephosphorylation through PP1 $\alpha / \gamma$ antagonism $^{25}$. MeV-V expression enhanced the Ser88 phosphorylation (indicative of ablated dephosphorylation) of GST-MDA5-2CARD or FLAG-MDA5 in a dose-dependent manner, as previously shown $^{25}$. Enhanced phosphorylation by $\mathrm{MeV}-\mathrm{V}$ correlated with a decline in ISGylation (Extended Data Fig. 3d,e). In contrast to WT MeV-V, a mutant MeV-V that has abolished PP1-binding and MDA5-dephosphorylation antagonism $(\mathrm{MeV}-\mathrm{V} \Delta \text { tail })^{25}$, exhibited little effect on MDA5-2CARD ISGylation (Extended Data Fig. 3f), strengthening the inhibition of ISGylation being primarily due to PP1 inhibition, and not other antagonistic effects, by MeV-V. The $\mathrm{V}$ proteins from Nipah and Hendra viruses (NiV-V and $\mathrm{HeV}-\mathrm{V}$ ) also enhanced MDA5 Ser88 phosphorylation and, correspondingly, dampened MDA5 ISGylation (Extended Data Fig. 3g,h), suggesting that several paramyxoviral V proteins inhibit MDA5 ISGylation through manipulation of Ser88 phosphorylation, although the precise mechanisms for individual $\mathrm{V}$ proteins remain to be determined. Taken together, these data suggest that the MDA5 CARD ISGylation is dependent on dephosphorylation at Ser88.

ISGylation promotes higher-order MDA5 assemblies. RLR activation requires RNA binding, RLR oligomerization and their translocation from the cytosol to mitochondria for an interaction with MAVS $^{5}$. To elucidate the mechanism by which ISGylation impacts MDA5 activity, we first examined whether ISGylation affects RNA binding. Endogenous MDA5 purified from WT or Isg15 $15^{-1-}$ MEFs interacted equally well with HMW-poly(I:C) in vitro (Extended Data Fig. 4a). MDA5 WT and Lys23Arg/Lys43Arg showed comparable binding to $\mathrm{HMW}$-poly(I:C), indicating that ISGylation does not affect the RNA-binding ability of MDA5 (Extended Data 
a

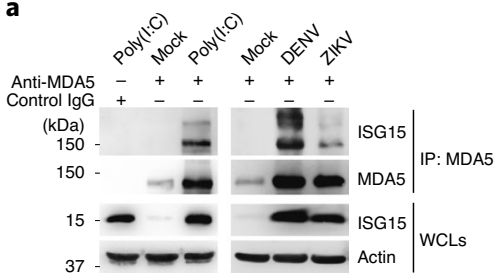

e
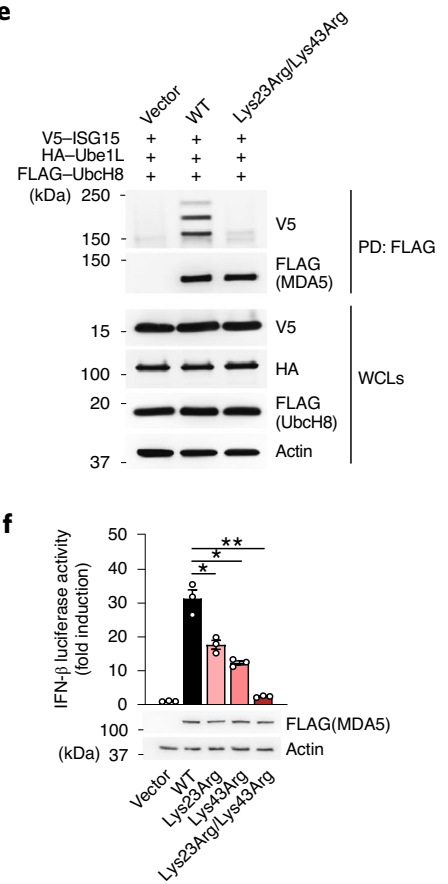

b

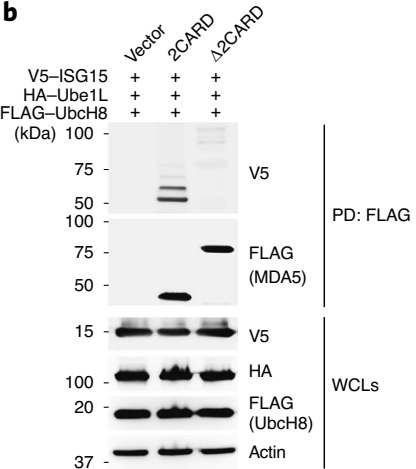

g
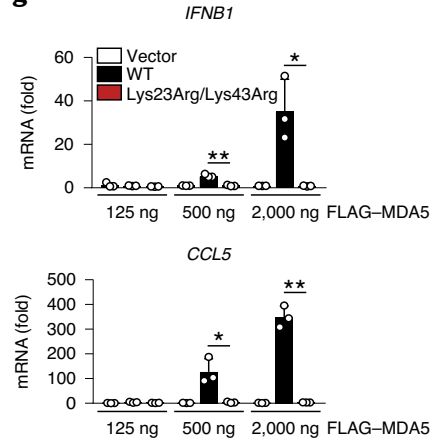

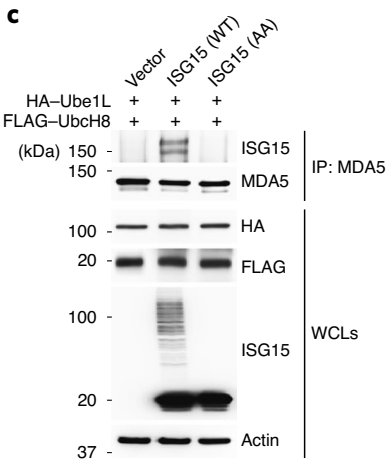

d

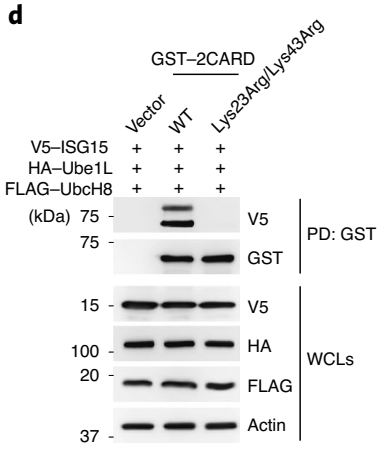

h

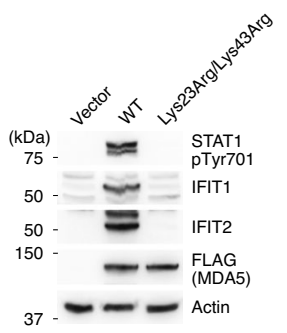

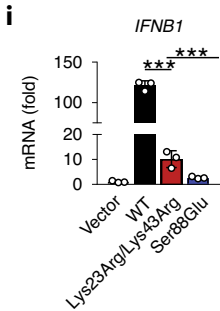
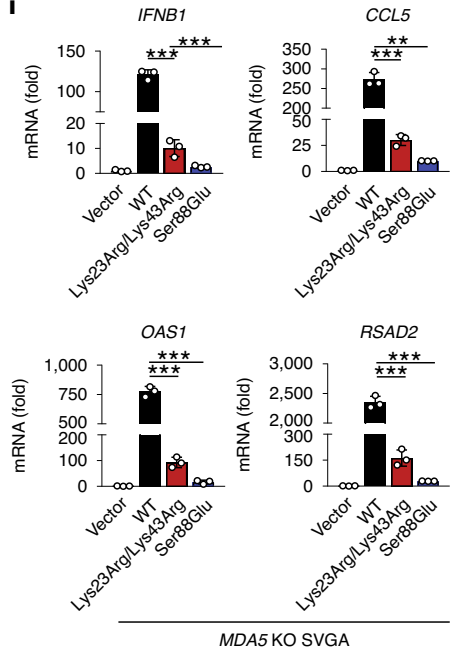

Fig. 2 | MDA5 activation requires ISGylation at Lys23 and Lys43. a, Endogenous MDA5 ISGylation in NHLFs that were mock treated, transfected with HMW-poly (I:C) $\left(0.1 \mu \mathrm{g} \mathrm{ml}^{-1}\right)$ for $40 \mathrm{~h}$ (left), or infected with DENV or ZIKV (MOI=1 for each) for $48 \mathrm{~h}$ (right), determined by IP with anti-MDA5 (or an IgG isotype control) and IB with anti-ISG15. b, ISGylation of FLAG-tagged MDA5-2CARD and MDA5 2CARD in transiently transfected HEK293T cells that also expressed V5-ISG15, HA-Ube1L and FLAG-UbcH8, assessed by FLAG PD and IB with anti-V5 at $40 \mathrm{~h}$ post-transfection. c, Endogenous MDA5 ISGylation in ISG15 KO HeLa cells stably reconstituted with vector, WT ISG15 or ISG15-AA and co-transfected with HA-Ube1L and FLAG-UbcH8 after IFN- $\beta$ treatment $\left(1,000 \mathrm{U} \mathrm{ml}^{-1}\right)$ for $24 \mathrm{~h}$, determined by IP with anti-MDA5 and IB with anti-ISG15. d, ISGylation of GST-MDA5-2CARD WT and Lys23Arg/ Lys43Arg in HEK293T cells that were co-transfected with V5-ISG15, HA-Ube1L and FLAG-UbcH8 for 24h, determined by GST PD and IB with anti-V5. e, ISGylation of FLAG-MDA5 WT and Lys23Arg/Lys43Arg in HEK293T cells that were co-transfected with V5-ISG15, HA-Ube1L and FLAG-UbcH8, determined by FLAG PD and IB with anti-V5. f, IFN- $\beta$ luciferase reporter activity in HEK293T cells that were transfected for 40 h with vector, FLAG-MDA5 WT or mutants. Luciferase values are presented as fold induction relative to the values for vector-transfected cells, set to 1 . WCLs were probed by IB with anti-FLAG and anti-actin. $\mathbf{g}$, RT-qPCR analysis of IFNB1 and CCL5 mRNA in HEK293T cells that were transiently transfected with either vector, or increasing amounts of FLAG-MDA5 WT or Lys23Arg/Lys43Arg. h, STAT1 phosphorylation and ISG (IFIT1 and -2) protein abundance in the WCLs of HEK293T cells that were transiently transfected with vector or FLAG-MDA5 WT or Lys23Arg/Lys43Arg, determined by IB. $\mathbf{i}$, RT-qPCR analysis of the indicated antiviral genes in MDA5 KO SVGAs that were transiently reconstituted with either empty vector or FLAG-tagged MDA5 WT, Lys23Arg/Lys43Arg or Ser88Glu. Data represent at least two independent experiments with similar results (mean \pm s.d. of $n=3$ biological replicates in $\mathbf{f}, \mathbf{g}$ and $\mathbf{i}$ ). ${ }^{\star} P<0.05,{ }^{\star \star} P<0.01$, ${ }^{\star \star \star} P<0.001$ (two-tailed, unpaired Student's $t$-test).

Fig. 4b). When we monitored the translocation of MDA5 from the cytosol to mitochondria following EMCV RNA stimulation, we found that ISG15 silencing, but not si.C transfection, abolished MDA5 translocation (Fig. 3a). In contrast, RIG-I translocation after $\mathrm{RABV}_{\mathrm{Le}}$ transfection was efficient in both ISG15-depleted and si.C-transfected cells (Fig. 3b). These data indicated that ISGylation regulates MDA5 translocation, or a step upstream of it. As the cytosol-to-mitochondria translocation of MDA5 requires an inter-

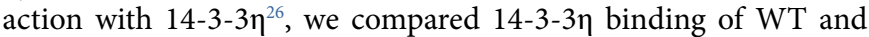
mutant MDA5. The ability of MDA5 Lys23Arg/Lys43Arg to bind 14-3-3ך was similar to that of WT MDA5 or the Lys68Arg mutant (Extended Data Fig. 4c). However, whereas EMCV RNA stimulation effectively induced MDA5 oligomerization in WT MEFs, the formation of MDA5 oligomers was ablated in ISG15-deficient MEFs (Fig. 3c). ISG15 knockdown in 293T cells also abolished the oligomerization of FLAG-MDA5-2CARD (Fig. 3d). Conversely, co-expression of the ISGylation machinery components, Ube1L and $\mathrm{UbcH} 8$, strongly enhanced MDA5-2CARD oligomerization in si.C-transfected cells, but not in ISG15-depleted cells (Fig. 3d), indicating that ISGylation is required for MDA5 oligomer formation. In support of this concept, FLAG-MDA5 Lys23Arg/Lys43Arg showed almost abolished oligomerization, whereas WT MDA5 oligomerized efficiently (Fig. 3e). We also compared the effect of the Lys23 Arg/Lys43Arg mutation with that of oligomerization-disruptive 

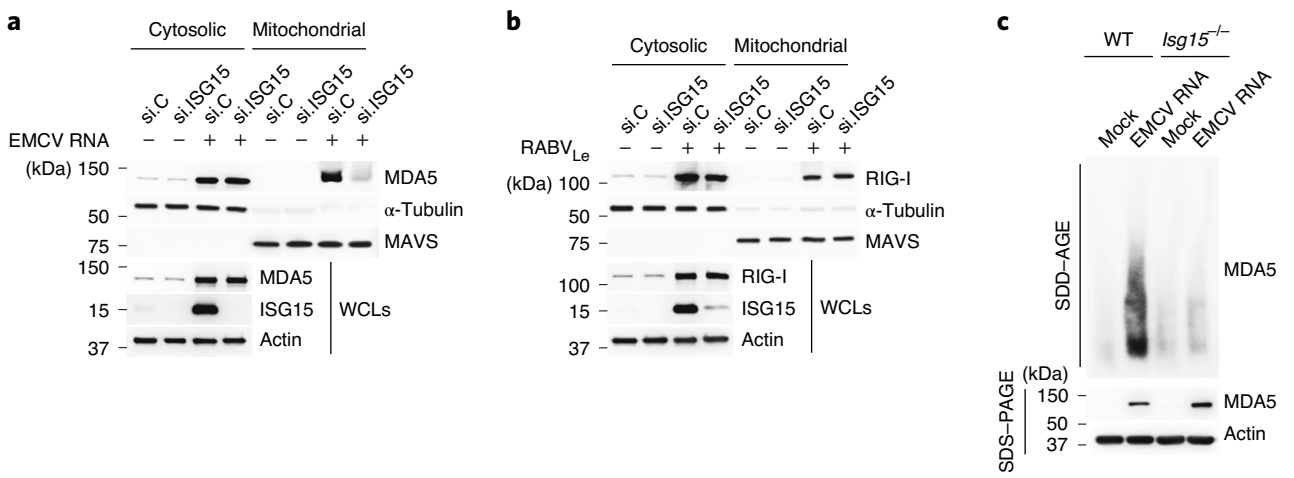

e

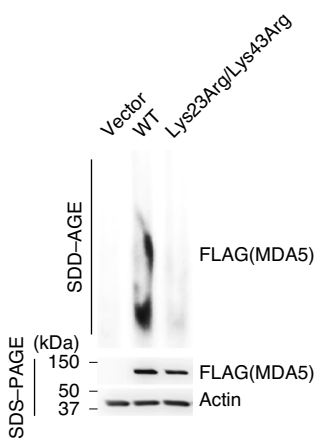

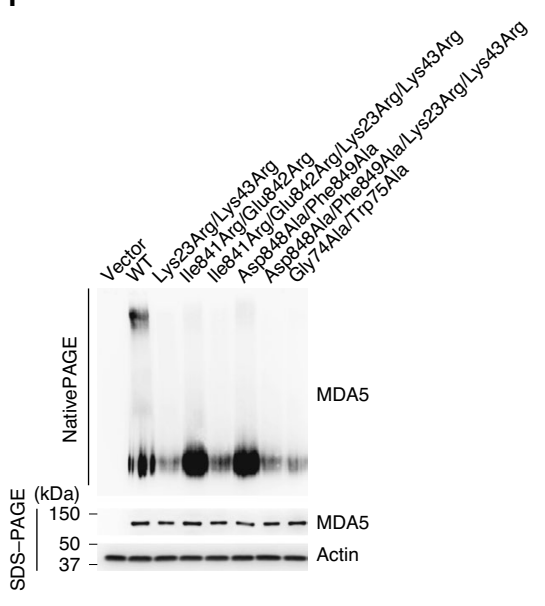

g

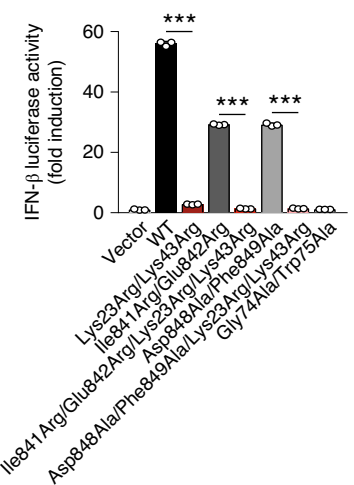

d

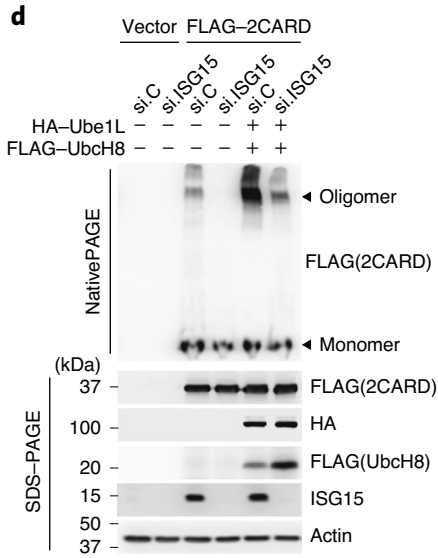

Fig. 3 | CARD ISGylation promotes the formation of higher-order MDA5 assemblies. a,b, Cytosol-mitochondria fractionation of WCLs from NHLFs that were transfected for $30 \mathrm{~h}$ with non-targeting control siRNA (si.C) or ISG15-specific siRNA (si.ISG15), and then mock treated or transfected with EMCV RNA $\left(0.4 \mu \mathrm{g} \mathrm{ml}^{-1}\right)(\mathbf{a})$ or RABV ${ }_{\mathrm{Le}}\left(1 \mathrm{pmol} \mathrm{ml}{ }^{-1}\right)(\mathbf{b})$ for $16 \mathrm{~h}$. IB was performed with anti-MDA5 (a), anti-RIG-I (b), anti-ISG15 and anti-actin (a and b). $\alpha$-Tubulin and MAVS served as purity markers for the cytosolic and mitochondrial fraction, respectively (a and $\mathbf{b}$ ). $\mathbf{c}$, Endogenous MDA5 oligomerization in WT and $/ \mathrm{sg} 15^{-1-}$ MEFs that were transfected with EMCV RNA $\left(0.5 \mu \mathrm{g} \mathrm{ml}^{-1}\right)$ for $16 \mathrm{~h}$, and assessed by SDD-AGE and IB with anti-MDA5. WCLs were further analysed by SDS-PAGE and probed by IB with anti-MDA5 and anti-actin. d, Oligomerization of FLAG-MDA5-2CARD in HEK293T cells that were transfected with the indicated siRNAs, either with or without HA-Ube1L and FLAG-UbcH8 for $48 \mathrm{~h}$, determined by NativePAGE and IB with anti-FLAG. WCLs were further analysed by SDS-PAGE and probed by IB with anti-FLAG, anti-HA, anti-ISG15 and anti-actin. e, Oligomerization of FLAG-MDA5 WT and Lys23Arg/Lys43Arg in transiently transfected MDA5 KO HEK293 cells, assessed by SDD-AGE and IB with anti-FLAG. WCLs were further analysed by SDS-PAGE and IB with anti-FLAG and anti-actin. f, Oligomerization of FLAG-tagged MDA5 WT and mutants in transiently transfected MDA5 KO HEK293 cells, assessed by NativePAGE and IB with anti-MDA5. WCLs were further analysed by SDS-PAGE and probed by IB with anti-MDA5 and anti-actin. $\mathbf{g}$, IFN- $\beta$ luciferase reporter activity in MDA5 KO HEK293 cells that were transfected for $24 \mathrm{~h}$ with either empty vector, or FLAG-tagged MDA5 WT or mutants. Luciferase activity is presented as fold induction relative to the values for vector-transfected cells, set to 1. Data represent at least two independent experiments with similar results (mean \pm s.d. of $n=3$ biological replicates in $\mathbf{g}$ ). ${ }^{\star \star \star} P<0.001$ (two-tailed, unpaired Student's $t$-test).

mutations that localize either to the interface between MDA5 monomers and impede RNA-binding-mediated MDA5 filamentation (Ile841Arg/Glu842Arg and Asp848Ala/Phe849Ala) ${ }^{27,28}$, or to the CARDs (Gly74Ala/Trp75Ala) and disrupt 2CARD oligomerization $^{27}$. Unlike WT MDA5, the Lys23Arg/Lys43Arg mutant, similar to MDA5 Gly74Ala/Trp75Ala, showed deficient oligomerization and, consistent with this, abolished IFN- $\beta$ promoter-activating ability (Fig. 3f,g). Introduction of Lys23Arg/Lys43Arg into the Ile841Arg/Glu842Arg or Asp848Ala/Phe849Ala background, either of which by itself decreased MDA5 oligomerization and signalling, also abolished MDA5 oligomer formation and IFN- $\beta$ induction (Fig. 3f,g). As LGP2 facilitates MDA5 nucleation on double-stranded RNA and thereby MDA5 oligomerization ${ }^{29,30}$, we compared LGP2 binding of MDA5 WT and Lys23Arg/Lys43Arg. MDA5 Lys23Arg/Lys43Arg interacted with LGP2 as efficiently as
WT MDA5 (Extended Data Fig. 4d), strengthening the proposal that CARD ISGylation promotes MDA5 oligomerization independently of RNA-binding-mediated filamentation. Collectively, these results establish that ISGylation facilitates CARD oligomerization and higher-order MDA5 assemblies.

ISGylation-dependent MDA5 signalling restricts virus replication. We next assessed whether ISGylation of MDA5 is required for its ability to restrict virus replication. FLAG-MDA5 WT, but not Lys23Arg/Lys43Arg, potently (by $\sim 2 \log$ ) inhibited EMCV replication (Fig. 4a). Similarly, MDA5 KO HEK293 cells reconstituted with WT MDA5, but not cells complemented with the Lys23Arg/ Lys43Arg mutant, effectively restricted DENV replication (Fig. 4b). We also reconstituted MDA5 KO astrocyte SVGAs, a physiologically relevant cell type for ZIKV infection, with either vector, or 
a
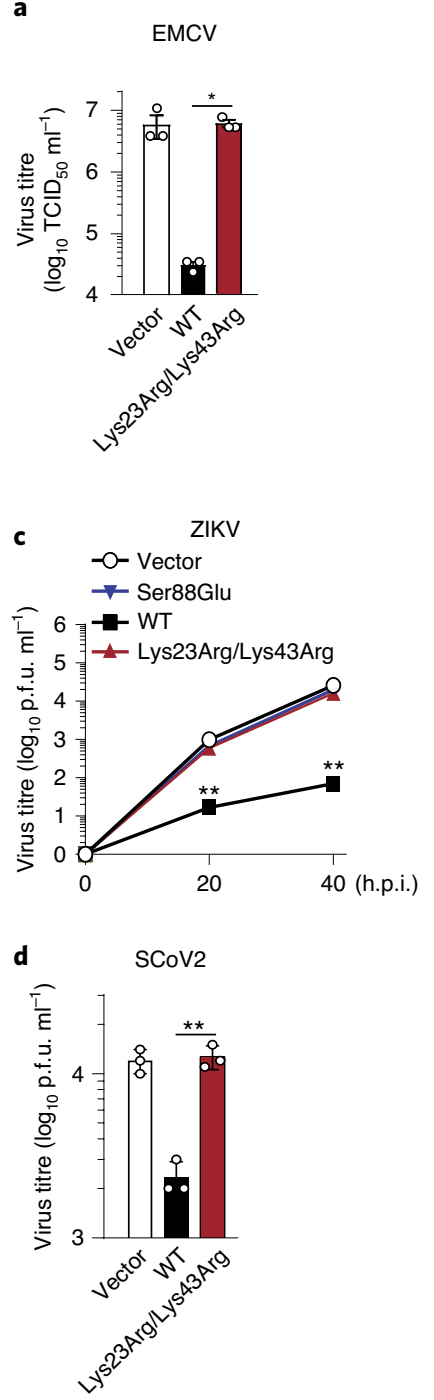

b

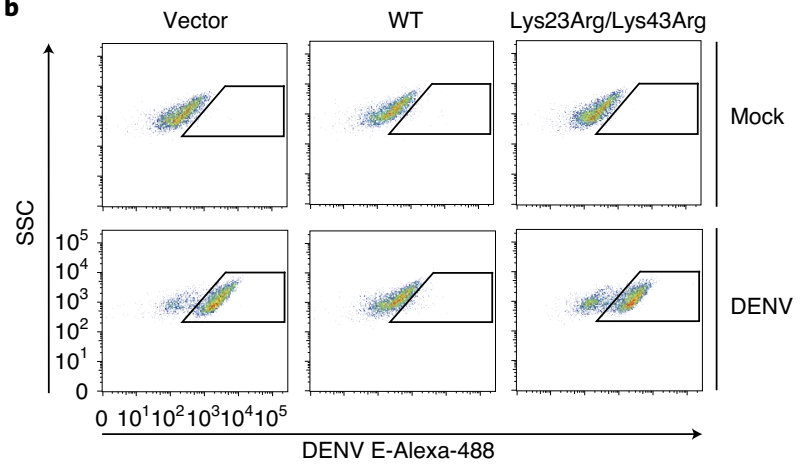

e $\quad$ si.C, si.ISG15

mutEMCV

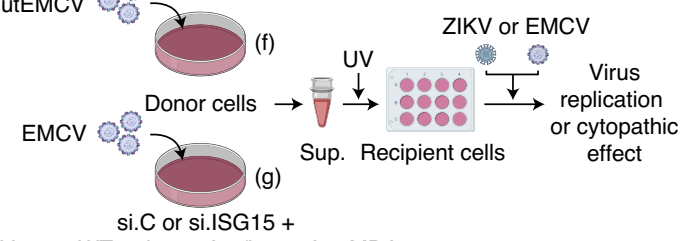

Vector, WT or Lys23Arg/Lys43Arg MDA5

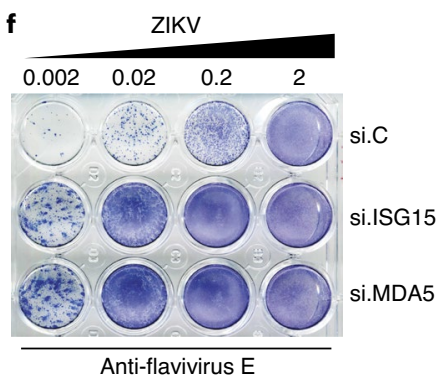

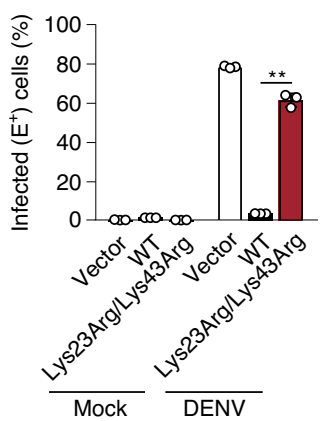

g

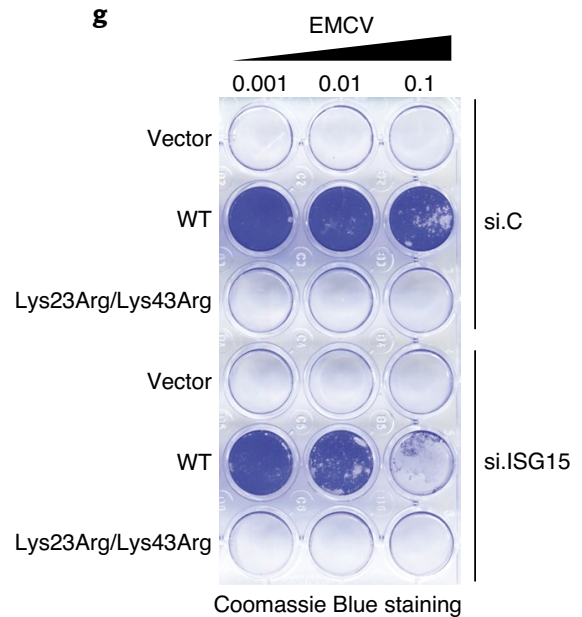

Fig. 4 | ISGylation is required for viral restriction by MDA5. a, EMCV titres in the supernatant of HEK293T cells that were transfected for 40 h with either vector, or FLAG-MDA5 WT or Lys23Arg/Lys43Arg, and then infected with EMCV (MOI=0.001) for 24h, determined by TCID 50 assay. b, Percentage of DENV-infected MDA5 KO HEK293 cells that were transfected for $24 \mathrm{~h}$ with either vector, or FLAG-MDA5 WT or Lys23Arg/Lys43Arg, and then mock treated or infected with DENV $(\mathrm{MOI}=5)$ for $48 \mathrm{~h}$, assessed by FACS using anti-flavivirus E (4G2). SSC, side scatter. c, ZIKV titres in the supernatant of MDA5 KO SVGAs that were transfected for $30 \mathrm{~h}$ with vector or FLAG-tagged MDA5 WT, Lys23Arg/Lys43Arg or Ser88Glu and then infected with ZIKV $(\mathrm{MOI}=0.1)$ for the indicated times, determined by plaque assay. p.f.u., plaque-forming units; h.p.i., hours post-infection. $\mathbf{d}$, SCoV2 titres in the supernatant of HEK293T-hACE2 cells that were transfected for $24 \mathrm{~h}$ with either empty vector, or FLAG-MDA5 WT or Lys23Arg/Lys43Arg, and then infected with SCoV2 (MOI = 0.5) for $24 \mathrm{~h}$, determined by plaque assay. e, Schematic of the experimental approach to 'decouple' the role of ISG15 in MDA5-mediated IFN induction from its role in dampening IFNAR signalling. Sup., supernatant. f, NHLF 'donor' cells were transfected for $40 \mathrm{~h}$ with the indicated siRNAs and then infected with mutEMCV $(\mathrm{MOI}=0.1)$ for $16 \mathrm{~h}$. Cell supernatants were UV inactivated and transferred on to Vero 'recipient' cells. After $24 \mathrm{~h}$, cells were infected with ZIKV (MOI =0.002-2) for 72 h, and ZIKV-positive cells determined by immunostaining with anti-flavivirus E (4G2) and TrueBlue peroxidase substrate. g, RIG-I KO HEK293 'donor' cells were transfected with si.C or si.ISG15 together with either vector, or FLAG-MDA5 WT or Lys23Arg/Lys43Arg, for 24h, followed by EMCV infection (MOI=0.001) for $16 \mathrm{~h}$. UV-inactivated cell supernatants were transferred on to Vero 'recipient' cells for $24 \mathrm{~h}$, followed by infection with EMCV (MOI=0.001-0.1) for $40 \mathrm{~h}$. EMCV-induced cytopathic effects were visualized by Coomassie Blue staining. Data represent at least two independent experiments with similar results (mean \pm s.d. of $n=3$ biological replicates in a-d). ${ }^{\star} P<0.05$, ${ }^{\star \star} P<0.01$ (two-tailed, unpaired Student's $t$-test).

MDA5 WT or Lys23Arg/Lys43Arg, and then assessed ZIKV replication over a $40-\mathrm{h}$ time course. ZIKV replication was attenuated by $\sim 100$-fold in cells reconstituted with WT MDA5 compared with vector-expressing cells. In contrast, cells complemented with MDA5 Lys23Arg/Lys43Arg did not restrict ZIKV, similar to cells expressing MDA5 Ser88Glu (Fig. 4c). WT MDA5, but not Lys23Arg/Lys43Arg, also restricted SCoV2 replication, although to a lesser extent than that seen for the other viruses tested (Fig. 4d).
We next determined the effect of ISG15 silencing on MDA5's ability to inhibit virus replication. Although MDA5 Lys23Arg/ Lys43Arg failed to suppress EMCV replication regardless of ISG15 silencing, WT MDA5 effectively restricted EMCV replication in si.C-transfected cells and, unexpectedly, also in ISG15-depleted cells (Extended Data Fig. 5a). In an exploration of the underlying mechanism of these unexpected results, we found that the EMCV-infected cells that expressed WT MDA5 had markedly enhanced levels of 
a

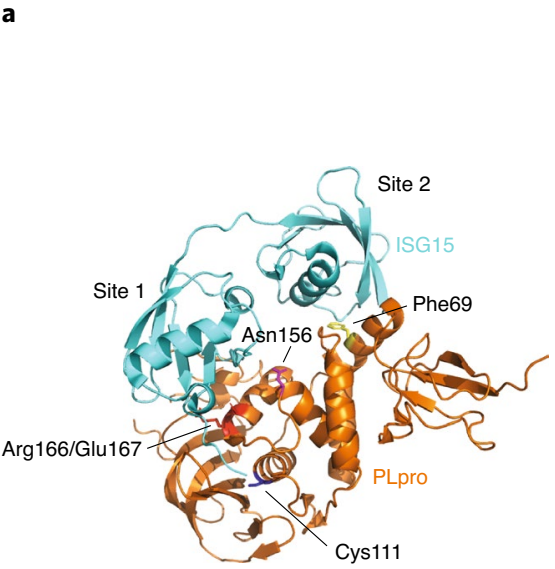

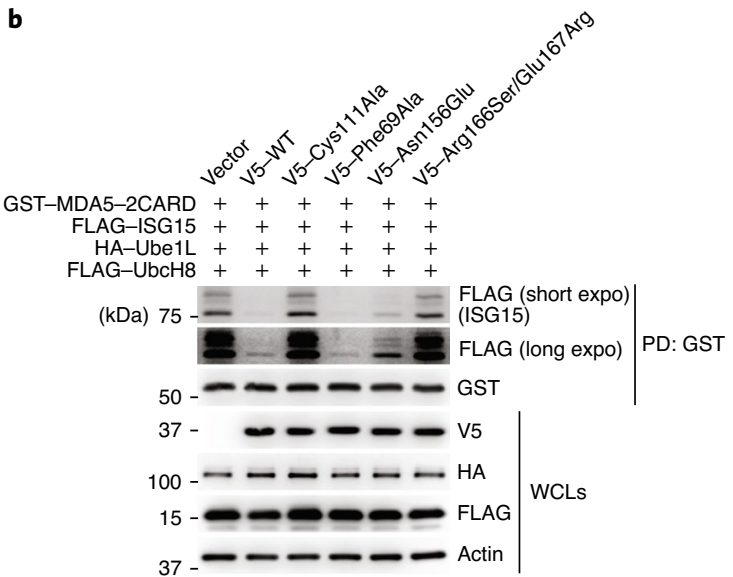

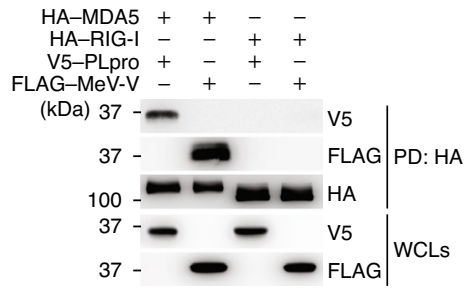

d

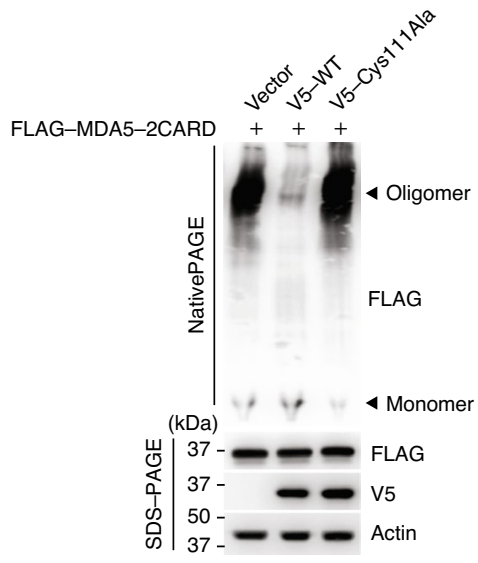

e

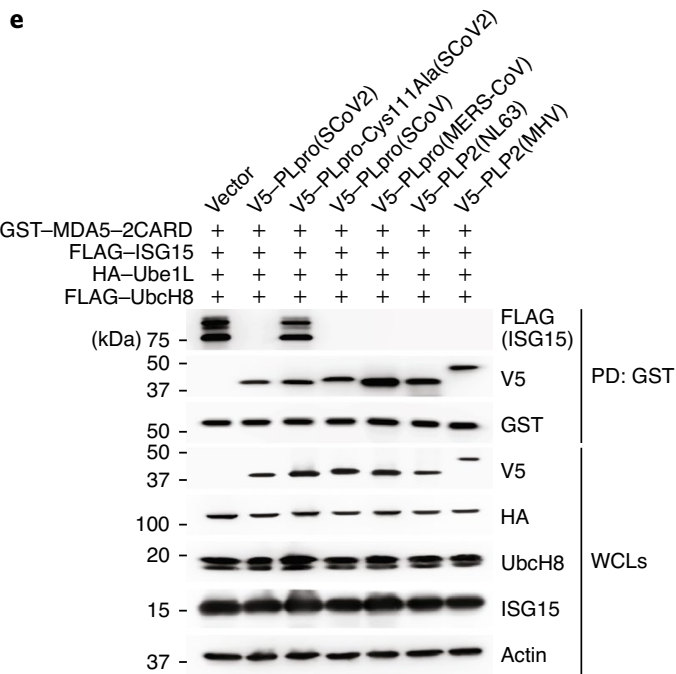

Fig. 5 | SCoV2 PLpro binds to and de-ISGylates MDA5-2CARD. a, Ribbon representation of the crystal structure of the SCoV2 PLpro:ISG15 complex (Protein Data Bank, accession no. 6YVA). Key residues that mediate site 1 interaction (Asn156 and Arg166/Glu167) or site 2 interaction (Phe69) in PLpro, as well as its catalytically active site (Cys111), are indicated. b, ISGylation of GST-MDA5-2CARD in HEK293T cells that were co-transfected for $20 \mathrm{~h}$ with vector or V5-tagged SCoV2 PLpro WT or mutants, along with FLAG-ISG15, HA-Ube1L and FLAG-UbcH8, determined by GST PD and IB with anti-FLAG and anti-GST. WCLs were probed by IB with anti-V5, anti-HA, anti-FLAG and anti-actin. c, Binding of HA-tagged MDA5 or RIG-I to V5-tagged SCoV2PLpro or FLAG-tagged MeV-V (positive control) in transiently transfected HEK293T cells, determined by HA PD and IB with anti-V5 or anti-FLAG, and anti-HA. WCLs were probed by IB with anti-V5 and anti-FLAG. d, Oligomerization of FLAG-MDA5-2CARD in HEK293T cells that were co-transfected with vector, or V5-tagged SCoV2 PLpro WT or Cys111Ala for $24 \mathrm{~h}$, assessed by NativePAGE and IB with anti-FLAG. WCLs were further analysed by SDSPAGE and probed by IB with anti-FLAG, anti-V5 and anti-actin. e, ISGylation of GST-MDA5-2CARD in HEK293T cells that also expressed FLAG-ISG15, HA-Ube1L and FLAG-UbcH8, and were co-transfected for $40 \mathrm{~h}$ with vector or the indicated V5-tagged coronaviral PLpro proteins, determined by GST PD and IB with anti-FLAG, anti-V5 and anti-GST. Data represent at least two independent experiments with similar results.

ISG protein expression when ISG15 was silenced compared with infected cells transfected with WT MDA5 and si.C (Extended Data Fig. 5b). Similarly, elevated ISG transcript and protein expression were observed in ISG15-deficient cells that were transfected with EMCV RNA or infected with mutEMCV, despite abrogation of IFN- $\beta$ induction (Extended Data Fig. $5 c, d$ ). In contrast, MDA5 knockdown abrogated both IFN- $\beta$ and ISG protein expression, as expected (Extended Data Fig. 5d). We noticed that the protein abundance of USP18, a deubiquitinating enzyme that negatively regulates IFNAR signaling ${ }^{31}$, was greatly diminished in ISG15-depleted cells following EMCV infection compared with infected cells that were transfected with si.C or MDA5-specific siRNA (Extended Data Fig. $5 \mathrm{~b}, \mathrm{~d})$, which is consistent with the reported role of ISG15 in preventing USP18 degradation ${ }^{32}$. Together, these data suggest that in experimental settings of ISG15-gene targeting (that is, silencing or KO) the antiviral effect of MDA5 ISGylation is masked by aberrant
ISG upregulation due to the ablation of ISG15's inhibitory effect on IFNAR signalling.

We next employed a virus protection assay that experimentally decouples MDA5 signalling in virus-infected cells from downstream IFNAR signalling in the same cells (Fig. 4e). Supernatants from mutEMCV-infected 'donor' cells that were either si.C transfected, or depleted of either ISG15 or MDA5, were ultraviolet (UV) inactivated and then transferred on to uninfected 'recipient' cells. 'Primed' recipient cells were then infected with ZIKV to directly monitor the antiviral effect of MDA5-mediated IFN production by donor cells. Whereas the supernatants from si.C-transfected 'donor' cells potently inhibited ZIKV replication, the supernatants from ISG15 or MDA5 knockdown cells minimally restricted ZIKV infection (Fig. 4f). Similarly, the culture supernatants from EMCV-infected donor cells transfected with WT MDA5 together with si.C led to greater protection of recipient cells from viral challenge than that 


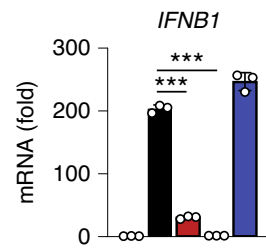

Mock RNA + - - -

SCoV2 RNA -++++

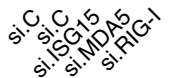

c

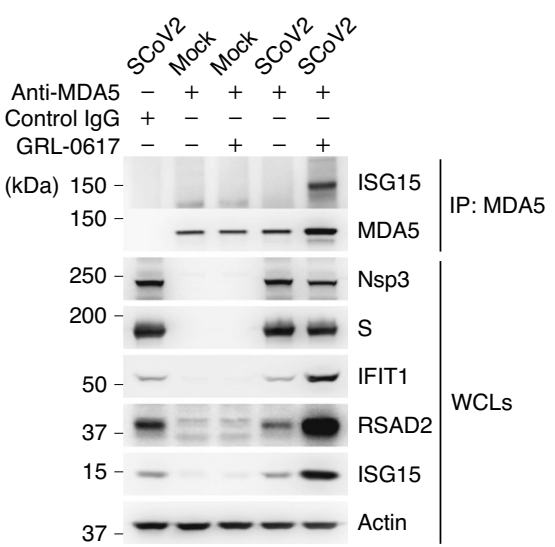

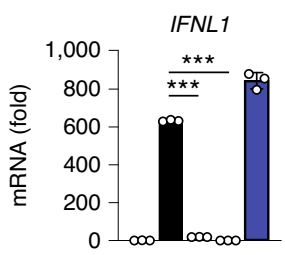

Mock RNA + - - -

SCOV2 RNA -++++

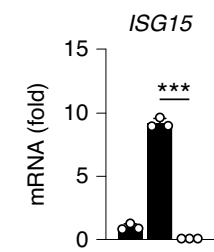

Mock RNA SCoV2 RNA -++ 5.

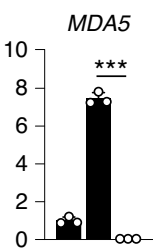

$+--$

$-++$ i. 200

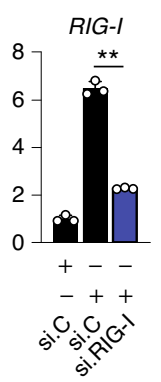

d
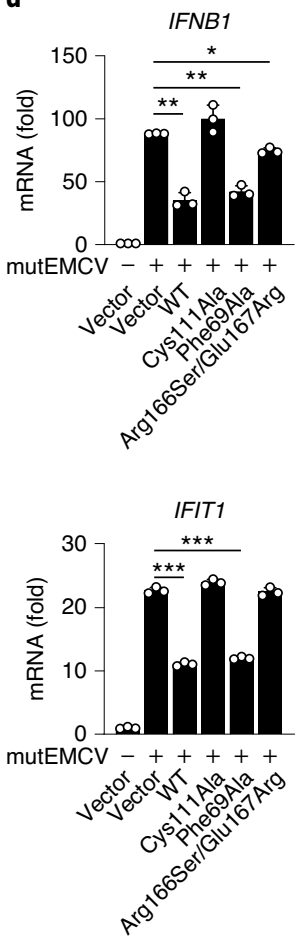
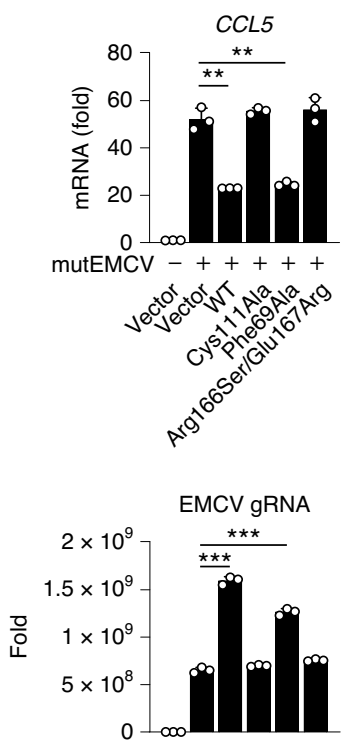

mutEMCV -+++++

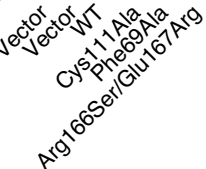

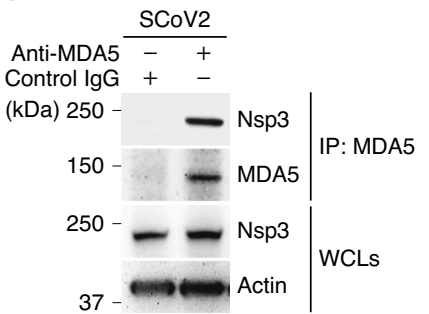

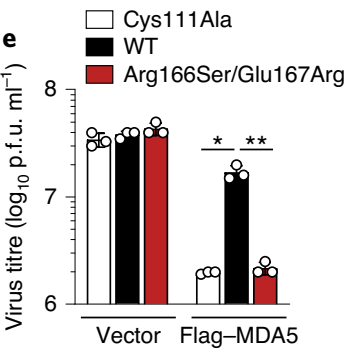

f

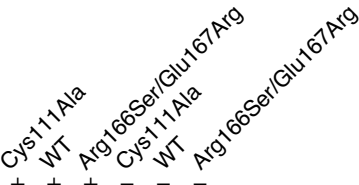

(kDa) 100 - $\quad-$ - FLAG
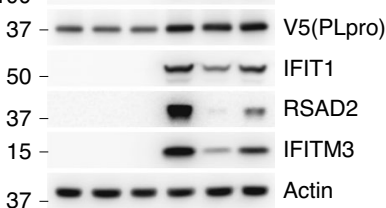

Fig. 6 | SCoV2 PLpro inhibits ISG15-mediated MDA5 signalling via its de-ISGylase activity. a, RT-qPCR analysis of IFNB1, IFNL1, ISG15, MDA5 and RIG-I transcripts in NHLFs that were transfected with the indicated siRNAs for $40 \mathrm{~h}$ and then transfected with mock RNA or SCoV2 RNA (0.4 $\mu$ g ml ${ }^{-1}$ ) for $24 \mathrm{~h}$. b. Binding of SCoV2 Nsp3 to endogenous MDA5 in A549-hACE2 cells that were infected with SCoV2 (MOI=0.5) for 24h, determined by IP with anti-MDA5 (or an IgG isotype control) followed by IB with anti-Nsp3 and anti-MDA5. WCLs were probed by IB with anti-Nsp3 and anti-actin. c, Endogenous MDA5 ISGylation in A549-hACE2 cells that were mock infected or infected with SCoV2 (MOI=0.5) for 40 h in the presence of PLpro inhibitor (GRL-0617; $50 \mu \mathrm{M}$ ) or vehicle control (dimethylsulfoxide), determined by IP with anti-MDA5 (or an IgG isotype control), followed by IB with anti-ISG15 and anti-MDA5. Protein abundance of IFIT1, RSAD2, ISG15 and actin in the WCLs was probed by IB. Efficient virus replication was verified by IB with anti-Nsp3 and anti-Spike (S). d, RT-qPCR analysis of IFNB1, CCL5 and IFIT1 transcripts, and EMCV genomic RNA (gRNA), in HeLa cells that were transiently transfected for $24 \mathrm{~h}$ with vector, or V5-SCoV2 PLpro WT or mutants, and then infected with mutEMCV (MOI=0.5) for $12 \mathrm{~h}$. e, EMCV titres in the supernatant of RIG-I KO HEK293 cells that were transiently transfected for 24 h with vector or FLAG-MDA5, along with V5-tagged SCoV2 PLpro WT, Cys111Ala or Arg166Ser/Glu167Arg, and then infected with EMCV (MOI=0.001) for 16 h, determined by plaque assay. f, Protein abundance of the indicated ISGs in the WCLs from the experiment in $\mathbf{e}$, determined by IB with the indicated antibodies. Data represent at least two independent experiments with similar results (mean \pm s.d. of $n=3$ biological replicates in a, d and e). ${ }^{\star} P<0.05,{ }^{\star \star} P<0.01$, ${ }^{\star \star \star} P<0.001$ (two-tailed, unpaired Student's $t$-test).

from cells expressing WT MDA5 and depleted of ISG15 (Fig. 4g). Collectively, these data demonstrate that ISGylation is important for MDA5-mediated restriction of a range of RNA viruses.

SCoV2 PLpro targets MDA5 for de-ISGylation. Coronaviruses such as SARS-CoV (SCoV), MERS-CoV and the recently emerged SCoV2 encode a PLpro that mediates viral polyprotein cleavage ${ }^{33}$. In addition, PLpro has deubiquitinating and de-ISGylating activities. SCoV2 PLpro was recently shown to modulate antiviral responses primarily via its de-ISGylase activity ${ }^{15}$. As MDA5 is known to be a major sensor for detecting coronaviruses ${ }^{34,35}$, and because our data showed that ISGylation is required for MDA5-mediated virus restriction, we examined whether SCoV2 PLpro enzymatically removes MDA5 ISGylation to antagonize innate immunity. SCoV2 PLpro WT, but not its catalytically inactive mutant (PLpro Cys111Ala) ${ }^{15}$, abolished the ISGylation of GST-MDA5-2CARD and FLAG-MDA5 (Fig. 5a,b and Extended Data Fig. 6a). The PLpro Asn156Glu and Arg166Ser/Glu167Arg mutants, which are marginally and severely impaired in ISG15 binding at the 'site 1' interface, respectively ${ }^{14,36}$, did slightly, or not, affect ISGylation. In contrast, PLpro Phe69Ala, in which the 'site 2' interface that preferentially determines binding to ubiquitin, but not ISG15, is disrupted ${ }^{14,36}$, 
diminished MDA5 ISGylation as potently as WT PLpro (Fig. 5a,b and Extended Data Fig. 6a). SCoV2 PLpro did not, however, suppress RIG-I-2CARD ubiquitination (Extended Data Fig. 6b).

We found that PLpro interacted specifically with MDA5, but not RIG-I, as did MeV-V which binds MDA5 and served as a control ${ }^{37}$ (Fig. 5c). Low amounts of PLpro inhibited signalling by MDA5, but not RIG-I, whereas higher amounts of PLpro suppressed antiviral signalling by both RLRs (Extended Data Fig. 6c). This strengthens MDA5 being a direct target of PLpro. De-ISGylation of IRF3 probably accounts for the inhibitory effect that higher doses of PLpro have on RLR signalling ${ }^{15,38}$.

When examining the effect of PLpro on MDA5-2CARD oligomerization, PLpro WT but not Cys111Ala efficiently blocked MDA5-2CARD oligomerization (Fig. 5d), indicating that SCoV2 PLpro inhibits the ISGylation-dependent MDA5 oligomer formation via its enzymatic activity.

The PLpro enzymes of the related $\beta$-coronaviruses, SCoV, MERS-CoV and murine hepatitis virus (MHV), as well as of the $\alpha$-coronavirus HCoV-NL63 (NL63) also bound to and efficiently reduced MDA5-2CARD ISGylation (Fig. 5e), suggesting that MDA5 antagonism by PLpro may be widely conserved among coronaviruses.

SCoV2 PLpro antagonizes ISG15-dependent MDA5 signalling. We next determined the relevance of ISG15-dependent MDA5 signalling for antiviral cytokine induction elicited by SCoV2. As SCoV2 infection is known to minimally induce type I IFNs due to effective viral antagonisms ${ }^{39}$, we isolated total RNA from SCoV2-infected cells and then re-transfected it into cells to stimulate innate immune signalling. SCoV2 RNA, but not RNA from mock-treated cells, robustly induced IFN transcripts; however, this induction was markedly diminished when ISG15 or MDA5 was silenced (Fig. 6a). RIG-I knockdown did not adversely affect the antiviral gene expression elicited by SCoV2 RNA, indicating that SCoV2 RNA-PAMPs are primarily sensed by the ISG15-MDA5 axis (Fig. 6a).

We found that SCoV2 non-structural protein 3 (Nsp3), within which PLpro lies, readily interacted with endogenous MDA5 during authentic SCoV2 infection (Fig. 6b). Endogenous MDA5 ISGylation was undetectable in SCoV2-infected cells, although the virus triggered ISG15 expression; however, in infected cells treated with a specific PLpro inhibitor ${ }^{15}$, MDA5 ISGylation and downstream ISG induction were strongly enhanced (Fig. 6c), supporting the proposal that PLpro effectively suppresses MDA5 ISGylation and signalling during live SCoV2 infection.

We next examined the effect of WT and mutant PLpro on the activation of endogenous MDA5 during mutEMCV infection. Consistent with their effect on MDA5 ISGylation (Fig. 5b and Extended Data Fig. 6a), SCoV2 PLpro WT and Phe69Ala prevented antiviral transcript induction, whereas MDA5 Arg166Ser/ Glu167Arg, similar to the Cys111Ala mutant, did not affect antiviral gene expression (Fig. 6d). In agreement with this, mutEMCV replication was enhanced in cells expressing PLpro WT or Phe69Ala, but not in cells expressing PLpro Cys111Ala or Arg166Ser/Glu167Arg (Fig. 6d). Likewise, WT PLpro, but not the Arg166Ser/Glu167Arg or Cys111Ala mutant, blocked EMCV restriction by FLAG-MDA5 (Fig. 6e); the effect on virus replication correlated with induced ISG proteins (Fig. 6f). Collectively, this establishes SCoV2 PLpro as an IFN antagonist that actively de-ISGylates MDA5.

\section{Discussion}

ISG15 conjugation is known to confer antiviral activity to a multitude of viruses; however, only few genuine substrates have been identified $^{12}$. On the other hand, ISG15 in its unconjugated form acts provirally by fortifying USP18-mediated IFNAR-signal inhibition $^{31,32,40}$. The present study identifies a key role for ISGylation in MDA5-mediated IFN induction. Our work also stresses the importance of experimental design in which decoupling the role of ISG15 in MDA5 activation from that in dampening IFNAR signalling is essential to reveal ISG15's potent antiviral activity. In an infected organism, it is probably the sum of multiple ISGylation events (affecting both host and viral proteins) that determines the outcome of infection and pathogenesis, which may be context dependent ${ }^{12}$.

Our findings indicate that ISGylation of MDA5 acts analogously to the Lys63-linked ubiquitination of RIG- ${ }^{5}$ : both PTMs (1) are regulated by PP1-induced dephosphorylation and (2) promote CARD oligomerization and RLR higher-order assemblies. However, whereas ubiquitin is abundant in both uninfected and infected cells, ISG15 expression is strongly increased by IFN stimulation. Nevertheless, even at basal levels, ISG15 is conjugated to many host proteins ${ }^{20}$, including MDA5 as our work showed, which may be sufficient for initial MDA5 activation. During viral infections that are sensed by multiple PRRs, MDA5 ISGylation may be a 'priming' mechanism whereby ISG15 upregulation by an immediate innate sensor (for example, RIG-I) ${ }^{41}$ primes MDA5 to enter a 'kick-start' mode. As ISG15 negatively regulates RIG-I ${ }^{16,17}$, ISGylation may trigger 'sensor switching' where MDA5 activation is promoted when ISG15 levels increase, while RIG-I activity is being dampened.

We identified that SCoV2 PLpro antagonizes MDA5 ISGylation via its enzymatic activity after binding to the sensor; this strategy is probably conserved among coronaviruses, which warrants further investigation. Cryo-electron microscopy analyses revealed that coronaviral Nsp3 is part of a pore complex that spans endoplasmic reticulum-derived double-membrane vesicles and exports newly synthesized viral $\mathrm{RNA}^{42}$. Thus, MDA5 may position itself in close proximity to the site of viral RNA export to facilitate PAMP detection; however, the PLpro domain of Nsp3 (which is on the cytoplasmic side) blocks MDA5 signalling through direct de-ISGylation. Some viruses may also inhibit MDA5 ISGylation through dysregulation of MDA5 phosphorylation, as shown for MeV-V.

In summary, our study uncovers a prominent role for ISGylation in activating MDA5-mediated immunity as well as its inhibition by SCoV2, unveiling a potential molecular target for the design of therapeutics against COVID-19.

\section{Methods}

Cell culture. HEK293T (human embryonic kidney cells), Vero (African green monkey kidney epithelial cells), BHK-21 (baby hamster kidney) and Aedes albopictus clone C6/36 cells were purchased from American Type Culture Collection (ATCC). Human PBMCs were isolated from unidentified healthy donor peripheral blood (HemaCare) and purified by Lymphoprep density gradient centrifugation (STEMCELL Technologies). The WT and isogenic Isg15 ${ }^{-/-} \mathrm{MEFs}$ were kindly provided by D. Lenschow (Washington University in St. Louis). SVGAs (human fetal glial astrocytes) were kindly provided by E. Cahir-McFarland (Biogen) ${ }^{43}$. SVGA MDA5 KO cells were generated by CRISPR (clustered regularly interspaced short palindromic repeats)-Cas9-mediated genome editing using a guide RNA (5'-AACTGCCTGCATGTTCCCGG-3') targeting the exon 1 of IFIH1/MDA5. The MDA5 KO and RIG-I KO HEK293 cells were a gift from J. Rehwinkel (University of Oxford) ${ }^{44}$. The WT and isogenic ISG15 KO HeLa cells were kindly provided by E. Schiebel (University of Heidelberg) ${ }^{45}$. ISG15 KO HeLa cells stably expressing FLAG-ISG15 WT or FLAG-ISG15-AA (GG156/157AA) were generated by lentiviral transduction followed by selection with puromycin $\left(2 \mu \mathrm{g} \mathrm{ml}^{-1}\right)$. HAP-1 WT and isogenic ISG15 KO cells were purchased from Horizon Discovery. HEK293T-hACE2 and Vero-E6-hACE2 cells were a gift from J. U. Jung (Cleveland Clinic Lerner Research Center). A549-hACE2 cells were kindly provided by B. R. tenOever (Icahn School of Medicine at Mount Sinai) ${ }^{39}$. HEK293T, HEK293, HeLa, MEF, NHLF, Vero, A549-hACE2 and BHK-21 cells were maintained in Dulbecco's modified Eagle's medium (DMEM, Gibco) supplemented with $10 \%$ (v:v) fetal bovine serum (FBS, Gibco), 2 mM GlutaMAX (Gibco), 1 mM sodium pyruvate (Gibco) and $100 \mathrm{U} \mathrm{ml}^{-1}$ of penicillin-streptomycin (Gibco). HEK293T-hACE2 and Vero-E6-hACE2 were maintained in DMEM containing $200 \mu \mathrm{g} \mathrm{ml}^{-1}$ of hygromycin B and $2 \mu \mathrm{g} \mathrm{ml}^{-1}$ of puromycin, respectively. SVGA and HAP-1 cells were cultured in Eagle's minimum essential medium (MEM, Gibco) and Iscove's modified Dulbecco's medium (Gibco), respectively, supplemented with $10 \% \mathrm{FBS}$ and $100 \mathrm{U} \mathrm{ml}^{-1}$ of penicillin-streptomycin. PBMCs were maintained in RPMI-1640 (Gibco) supplemented with $10 \%$ FBS and $100 \mathrm{U} \mathrm{ml}^{-1}$ of penicillinstreptomycin. C6/36 cells were cultured in MEM with $10 \% \mathrm{FBS}$ and $100 \mathrm{U} \mathrm{ml}^{-1}$ of 
penicillin-streptomycin. Except for C6/36 cells that were maintained at $28^{\circ} \mathrm{C}$, all cell cultures were maintained at $37^{\circ} \mathrm{C}$ in a humidified $5 \% \mathrm{CO}_{2}$ atmosphere.

Commercially obtained cell lines were authenticated by vendors and were not validated further in our laboratory. Cell lines that were obtained and validated by other groups were not further authenticated. KO cell lines were validated by confirming the absence of target protein expression. All cell lines used in the present study have been regularly tested for potential Mycoplasma contamination by PCR or using the MycoAlert Kit (Lonza).

Viruses. DENV (serotype 2, strain 16681) and ZIKV (strain BRA/Fortaleza/2015) were propagated in C6/36 and Vero cells, respectively ${ }^{46,47}$. EMCV (EMC strain) was purchased from ATCC and propagated in HEK293T cells ${ }^{6}$; mutEMCV $\left(\mathrm{EMCV}-\mathrm{Zn}_{\mathrm{C} 19 \mathrm{~A} / \mathrm{C22A} \mathrm{A}}\right)$, which carries two point mutations in the zinc domain of the L protein ${ }^{18}$, was kindly provided by F. J. M. van Kuppeveld (Utrecht University) and was propagated in BHK-21 cells. Sendai virus (strain Cantell) was purchased from Charles River Laboratories. SCoV2 (strain 2019-nCoV/USA WA1/2020) was kindly provided by J. U. Jung (Cleveland Clinic Lerner Research Center) and was propagated in Vero-E6-hACE2 cells. All work relating to SCoV2 live virus and SCoV2 RNA was conducted in the BSL-3 facility of the Cleveland Clinic Florida Research and Innovation Center in accordance with institutional biosafety committee regulations.

DNA constructs and transfection. The human MDA5 open reading frame (ORF) containing an N-terminal FLAG tag was amplified from the pEF-BosFLAG-MDA5 (ref. ${ }^{6}$ ) and subcloned into pcDNA3.1/Myc-His B between Xhol and AgeI. Site-directed mutagenesis on pcDNA3.1-FLAG-MDA5 (Lys23Arg/ Lys43Arg, Ser88Ala, Ser88Glu, Ile841Arg/Glu842Arg, Asp848Ala/Phe849Ala and Gly74Ala/Trp75Ala) was introduced by overlapping PCR. HA-MDA5 was cloned into pcDNA3. $1^{+}$between KpnI and XhoI. GST-MDA5-2CARD (in a pEBG vector) and its Ser88Ala, Ser88Asp and Ser88Glu derivatives have been described previously ${ }^{6}$. The single (Lys23Arg, Lys43Arg, Lys68Arg, Lys128Arg, Lys137Arg, Lys169Arg, Lys174Arg and Lys235Arg) and double (Lys23Arg/ Lys43Arg) mutations of MDA5-2CARD (amino acids 1-295) were introduced by site-directed mutagenesis into GST-MDA5-2CARD. In addition, MDA5-2CARD and its Lys23Arg/Lys43Arg mutant were subcloned into pcDNA3.1- harbouring an N-terminal $3 \times$ FLAG tag between NheI and NotI. The pCR3-FLAG-MV-V (strain Schwarz) was a gift from K.-K. Conzelmann (LMU, Munich); pEF-BosFLAG-NiV-V, pCAGGS-HA-MeV-V and pCAGGS-HA-MeV-V $\Delta$ tail have been described previously ${ }^{25}$. PIV2-V, PIV5-V, MenV-V, MPRV-V and HeV-V constructs were kindly provided by S. Goodbourn (University of London), and the respective ORF was subcloned into pEF-Bos containing an N-terminal FLAG tag between NotI and SalI. The pEF-Bos-FLAG-MuV-V was a gift from C. Horvath (Addgene, catalogue no. 44908 (ref. ${ }^{48}$ )); pCAGGS-V5-hISG15 was a gift from A. García-Sastre (Icahn School of Medicine at Mount Sinai) ${ }^{49}$; pCAGGS-HA-Ube1L and pFLAG-CMV2-UbcH8 were kindly provided by J. U. Jung (University of Southern California); pcDNA3.1-Myc-UBE2I was cloned by ligating a synthetic UBE2I ORF into pcDNA3.1/Myc-His B between HindIII and NotI. FLAG-SUMO1 was obtained from F. Full (University of Erlangen-Nuremberg). V5-tagged SARS-CoV-PLpro, MERS-CoV-PLpro, NL63-PLP2 and MHV-PLP2 in pcDNA3.1-V5/His B were kindly provided by S. C. Baker (Loyola University of Chicago). The SARS-CoV-2 PLpro ORF (amino acids 746-1,060) was amplified from pDONR207 SARS-CoV-2 NSP3 (a gift from F. Roth; Addgene catalogue no. 141257 (ref. $\left.{ }^{50}\right)$ ) and subcloned into pcDNA3.1-V5. The Cys111Ala, Phe69Ala, Asn156Glu and Arg166Ser/Glu167Arg mutations of SARS-CoV-2 PLpro were introduced by site-directed mutagenesis. The correct sequence of all constructs was confirmed by DNA sequencing. Transient DNA transfections were performed using linear poly(ethylenimine) $\left(1 \mathrm{mg} \mathrm{ml}^{-1}\right.$ of solution in $10 \mathrm{mM}$ Tris- $\mathrm{HCl}, \mathrm{pH}$ 6.8; Polysciences), Lipofectamine 2000 (Invitrogen), Lipofectamine LTX with Plus Reagent (Invitrogen), TransIT-HeLaMONSTER (Mirus) or TransIT-X2 Transfection Reagent (Mirus) as per the manufacturers' instructions.

Antibodies and other reagents. Primary antibodies used in the present study include anti-GST (1:5,000; Sigma-Aldrich), anti-V5 (1:5,000, R960-25; Novex), anti-FLAG (M2, 1:2,000; Sigma-Aldrich), anti-HA (1:3,000, HA-7; Sigma-Aldrich), anti-Phospho-IRF3 (Ser396) (1:1,000, D6O1M; CST), anti-IRF3 (1:1,000, D6I4C; CST), anti-Phospho-STAT1 (Tyr701) (1:1,000, 58D6; CST), anti-IFIT1 (1:1,000, PA3-848; Invitrogen and 1:1,000, D2X9Z; CST), anti-IFIT2 (1:1,000; Proteintech), anti-ISG15 (1:500, F-9; Santa Cruz), anti-MAVS (1:1,000; CST), anti-RIG-I (1:2,000, Alme-1; Adipogen), anti-MDA5 (1:1,000, D74E4; CST), anti-Phospho-MDA5 (Ser88) ${ }^{6}$, anti-PP1 $\alpha$ (1:2,000; Bethyl laboratories), anti-PP1 $\gamma$ (1:2,000; Bethyl laboratories), anti-USP18 (1:1000, D4E7; CST), anti-RSAD2 (1:1,000, D5T2X; CST), anti-PKR (1:1,000, D7F7; CST), anti-MX1 (1:1,000, D3W7I; CST), anti-IFITM3 (1:1,000, D8E8G; CST), anti-ISG20 (1:1,000, PA5-30073; Invitrogen), anti-ubiquitin (1:1,000, P4D1; Santa Cruz), anti-NS3 (ref. ${ }^{47}$ ), anti-Nsp3 (1:1,000, GTX135589; GeneTex), anti-Spike (1:1,000, 1A9; GeneTex), anti- $\alpha$-tubulin (1:1,000; CST) and anti- $\beta$-actin $(1: 1,000, \mathrm{C} 4)$. Monoclonal anti-MDA5 antibody was purified from mouse hybridoma cell lines kindly provided by J. Rehwinkel (University of Oxford) ${ }^{44}$. Monoclonal anti-IFNAR2-neutralizing antibody (1:250, MMHAR-2) was obtained from PBL
Assay Science. Monoclonal anti-flavivirus E antibody (4G2) was purified from the mouse hybridoma cell line D1-4G2-4-15 (ATCC). Anti-mouse and anti-rabbit horseradish peroxidase-conjugated secondary antibodies $(1: 2,000)$ were purchased from CST. Anti-FLAG M2 magnetic beads (MilliporeSigma), anti-FLAG agarose beads (MilliporeSigma), Glutathione Sepharose 4B resin (GE Healthcare) and Protein G Dynabeads (Invitrogen) were used for protein IP. Protease and phosphatase inhibitors were obtained from MilliporeSigma. HMW-poly(I:C)/ LyoVec and HMW-poly(I:C) biotin were obtained from Invivogen. Human IFN- $\beta$ was purchased from PBL Biomedical Laboratories. GRL-0617 was purchased from AdooQ Bioscience.

Mass spectrometry. Large-scale GST pulldown (PD) and MS analysis were performed as previously described ${ }^{7,22}$. Briefly, HEK293T cells were transfected with GST or GST-MDA5-2CARD, and the cells were collected at $48 \mathrm{~h}$ post-transfection and lysed in Nonidet P-40 (NP-40) buffer (50 mM 4-(2-hydroxyethyl)1-piperazine-ethanesulfonic acid (Hepes), pH 7.4, $150 \mathrm{mM} \mathrm{NaCl}, 1 \%$ (v:v) NP-40, $1 \mathrm{mM}$ ethylenediaminetetraacetic acid (EDTA) and $1 \times$ protease inhibitor cocktail (MilliporeSigma)). Cell lysates were cleared by centrifugation at $16,000 \mathrm{~g}$ and $4{ }^{\circ} \mathrm{C}$ for $20 \mathrm{~min}$, and cleared supernatants were subjected to GST PD using Glutathione Sepharose $4 \mathrm{~B}$ beads (GE Healthcare) at $4^{\circ} \mathrm{C}$ for $4 \mathrm{~h}$. The beads were extensively washed with NP-40 buffer and proteins eluted by heating in $1 \times$ Laemmli sodium dodecylsulfate (SDS) sample buffer at $95^{\circ} \mathrm{C}$ for $5 \mathrm{~min}$. Eluted proteins were resolved on a NuPAGE $4-12 \%$ Bis-Tris gel (Invitrogen) and then stained at room temperature using the SilverQuest Silver Staining Kit (Invitrogen). The bands that were specifically present in the GST-MDA5-2CARD sample, but not the GST control sample, were excised and analysed by LC-MS/MS (Taplin Mass Spectrometry Facility, Harvard University).

Immunoprecipitation and immunoblotting. Cells were transfected with FLAG-MDA5, GST-MDA5-2CARD or FLAG-MDA5-2CARD in the absence or presence of ISGylation machinery components (that is, HA-Ube1L, FLAGUbcH8 and V5-ISG15) as indicated. After $48 \mathrm{~h}$, cells were lysed in NP-40 buffer and cleared by centrifugation at $16,000 \mathrm{~g}$ and $4{ }^{\circ} \mathrm{C}$ for $20 \mathrm{~min}$. Cell lysates were then subjected to GST or FLAG PD using glutathione magnetic agarose beads (Pierce) and anti-FLAG M2 magnetic beads (MilliporeSigma) at $4{ }^{\circ} \mathrm{C}$ for $4 \mathrm{~h}$ or $16 \mathrm{~h}$, respectively. The beads were extensively washed with NP-40 buffer and proteins eluted by heating in $1 \times$ Laemmli SDS sample buffer at $95^{\circ} \mathrm{C}$ for $5 \mathrm{~min}$ or by competition with FLAG peptide (MilliporeSigma) at $4^{\circ} \mathrm{C}$ for $4 \mathrm{~h}$. For endogenous MDA5 IP, NHLFs were stimulated with HMW-poly(I:C)/LyoVec $\left(0.1 \mu \mathrm{g} \mathrm{ml}^{-1}\right)$ or infected with DENV or ZIKV at the indicated multiplicity of infection (MOI) for $40 \mathrm{~h}$. Cell lysates were precleared with Protein G Dynabeads (Invitrogen) at $4{ }^{\circ} \mathrm{C}$ for $2 \mathrm{~h}$, and then incubated with Protein G Dynabeads conjugated with the anti-MDA5 antibody or an immunoglobulin (Ig)G1 isotype control (G3A1; CST) at $4{ }^{\circ} \mathrm{C}$ for $4 \mathrm{~h}$. The beads were washed four times with RIPA buffer (20 mM Tris-HCl, pH 8.0, $150 \mathrm{mM} \mathrm{NaCl}, 1 \%$ (v:v) NP-40, 1\% (w:v) deoxycholic acid and $0.01 \%(\mathrm{w}: \mathrm{v})$ SDS) and protein eluted in $1 \times$ Laemmli SDS sample buffer. Protein samples were resolved on Bis-Tris SDS-polyacrylamide gel electrophoresis (PAGE) gels, transferred on to polyvinylidene difluoride (PVDF) membranes (Bio-Rad), and visualized using the SuperSignal West Pico PLUS or Femto chemiluminescence reagents (Thermo Fisher Scientific) on an ImageQuant LAS 4000 Chemiluminescent Image Analyzer (General Electric) as previously described ${ }^{47}$

Enzyme-linked immunosorbent assay (ELISA). Human or mouse IFN- $\beta$ in the culture supernatants of NHLFs, HeLa and MEFs was determined by ELISA using the VeriKine Human Interferon Beta ELISA Kit or VeriKine Mouse Interferon Beta ELISA Kit (PBL Assay Science) as previously described ${ }^{6}$

Knockdown mediated by siRNA and shRNA. Transient knockdown in NHLFs, HeLa, HAP-1, HEK293T and HEK293 cells was performed using non-targeting or gene-specific siGENOME SMARTpool small interfering (si)RNAs (Horizon Discovery). These are the Non-Targeting siRNA Pool no. 2 (D-001206-14), IFIH1 (M-013041-00), DDX58 (M-012511-01), PPP1CA (M-008927-01), PPP1CC (M-006827-00) and ISG15 (D-004235-17 and D-004235-18). Transfection of siRNAs was performed using the Lipofectamine RNAiMAX Transfection Reagent (Invitrogen) as per the manufacturer's instructions. Scrambled short hairpin (sh) RNA control lentiviral particles and shRNA lentiviral particles targeting ISG15 (TL319471V) or IFIH1 (TL303992V) were purchased from OriGene. Lentiviral transduction of human PBMCs $\left(1 \times 10^{5}\right.$ cells; MOI $\left.=8\right)$ was performed in the presence of $8 \mu \mathrm{g} \mathrm{ml}^{-1}$ of polybrene (Santa Cruz). Knockdown efficiency was determined by quantitative real-time PCR (RT-qPCR) or IB as indicated.

RT-qPCR. Total RNA was purified using the E.Z.N.A. HP Total RNA Kit (Omega Bio-tek) as per the manufacturer's instructions. One-step RT-qPCR was performed using the SuperScript III Platinum One-Step qRT-PCR Kit (Invitrogen) and predesigned PrimeTime qPCR Probe Assays (Integrated DNA Technologies) on a 7500 Fast Real-Time PCR System (Applied Biosystems). Relative mRNA expression was normalized to the levels of $G A P D H$ and expressed relative to the values for control cells using the $\Delta \Delta C_{\mathrm{t}}$ method 
Luciferase reporter assay. IFN- $\beta$ reporter assay was performed as previously described $^{51}$. Briefly, HEK293T or MDA5 KO HEK293 cells were transfected with IFN- $\beta$ luciferase reporter construct and $\beta$-galactosidase ( $\beta$-gal) expressing pGK- $\beta$-gal, along with GST-MDA5-2CARD (WT or mutants) or FLAG-MDA5 (WT or mutants). At the indicated time points after transfection, luciferase and $\beta$-gal activities were determined using, respectively, the Luciferase Assay System (Promega) and $\beta$-Galactosidase Enzyme Assay System (Promega) on a Synergy HT microplate reader (BioTek). Luciferase activity was normalized to $\beta$-gal values, and fold induction was calculated relative to vector-transfected samples, set to 1 .

Cytosol-mitochondria fractionation assay. The cytosol-mitochondria fractionation assay was performed using a Mitochondria/Cytosol Fractionation Kit (Millipore) as previously described ${ }^{46,47}$. Briefly, NHLFs were transfected for $30 \mathrm{~h}$ with either non-targeting control siRNA or ISG15-specific siRNA and then transfected with EMCV RNA or RABV $\mathrm{Le}_{\mathrm{L}}$ for $16 \mathrm{~h}$. Cells were homogenized in an isotonic buffer using a Dounce homogenizer and the lysates were centrifuged at $600 \mathrm{~g}$ to pellet the nuclei and unbroken cells. The supernatant was further centrifuged at $10,000 \mathrm{~g}$ and $4{ }^{\circ} \mathrm{C}$ for $30 \mathrm{~min}$ to separate the cytosolic (supernatant) and mitochondrial (pellet) fractions. The protein concentration of both fractions was determined by a bicinchoninic acid assay (Pierce), and equal amounts of proteins were analysed by IB. Anti- $\alpha$-tubulin and anti-MAVS IB served as markers for the cytosolic and mitochondrial fractions, respectively.

In vitro RNA-binding assay. WT and $I s g 15^{-/-}$MEFs were stimulated with IFN- $\beta$ $\left(1,000 \mathrm{U} \mathrm{ml}^{-1}\right)$ for $24 \mathrm{~h}$. Cells were lysed in a buffer containing $50 \mathrm{mM}$ Hepes, $\mathrm{pH} 7.4,200 \mathrm{mM} \mathrm{NaCl}, 1 \%$ (v:v) NP-40, $1 \mathrm{mM}$ EDTA and $1 \times$ protease inhibitor cocktail (MilliporeSigma). NeutrAvidin agarose beads (Pierce) were conjugated with the biotinylated $\mathrm{HMW}$-poly(I:C) at $4^{\circ} \mathrm{C}$ for $4 \mathrm{~h}$. Cell lysates were incubated with the conjugated beads at $4^{\circ} \mathrm{C}$ for $16 \mathrm{~h}$. The beads were washed three times with lysis buffer and then boiled at $95^{\circ} \mathrm{C}$ in $1 \times$ Laemmli SDS sample buffer to elute the proteins. Precipitated proteins were resolved on Bis-Tris SDS-PAGE gels and analysed by IB with anti-MDA5. Equal input MDA5 protein amounts were confirmed by IB with anti-MDA5.

NativePAGE. NativePAGE for analysing endogenous IRF3 dimerization was performed as previously described ${ }^{52}$. For measuring MDA5 oligomerization, HEK293T or MDA5 KO HEK293 cells were transfected with WT or mutant FLAG-MDA5-2CARD or FLAG-MDA5 as indicated. Cells were lysed in 1× native PAGE sample buffer (Invitrogen) containing 1\% (v:v) NP-40 on ice for $30 \mathrm{~min}$, and then lysates were cleared by centrifugation at $16,000 \mathrm{~g}$ and $4{ }^{\circ} \mathrm{C}$ for $10 \mathrm{~min}$. Cleared lysates were resolved on a $3-12 \%$ Bis-Tris NativePAGE gel (Invitrogen) as per the manufacturer's instructions and analysed by IB with the indicated antibodies.

Semi-denaturating detergent agarose gel electrophoresis. MDA5 oligomerization in MEFs transfected with EMCV RNA, or in MDA5 KO HEK293 cells reconstituted with WT or mutant FLAG-MDA5, was determined by semi-denaturating detergent agarose gel electrophoresis (SDD-AGE) as previously described with modifications ${ }^{26}$. Briefly, cells were lysed in a buffer containing $50 \mathrm{mM}$ Hepes, pH 7.4, $150 \mathrm{mM} \mathrm{NaCl}, 0.5 \%$ (v:v) NP-40, $10 \%$ (v:v) glycerol and $1 \times$ protease inhibitor cocktail (MilliporeSigma) at $4^{\circ} \mathrm{C}$ for $20 \mathrm{~min}$. Cell lysates were cleared by centrifugation at $16,000 \mathrm{~g}$ and $4{ }^{\circ} \mathrm{C}$ for $10 \mathrm{~min}$ and then incubated on ice for $1 \mathrm{~h}$. Cell lysates were subsequently incubated in $1 \times$ SDD-AGE buffer $(0.5 \times$ Tris/borate/EDTA (TBE), $10 \%$ (v:v) glycerol, and $2 \%$ (w:v) SDS) for $15 \mathrm{~min}$ at room temperature and resolved on a vertical $1.5 \%$ agarose gel containing $1 \times \mathrm{TBE}$ and $0.1 \%(\mathrm{w}: \mathrm{v}) \mathrm{SDS}$ at $80 \mathrm{~V}$ for $90 \mathrm{~min}$ at $4{ }^{\circ} \mathrm{C}$. Proteins were transferred on to a PVDF membrane and analysed by IB with the indicated antibodies.

Viral RNA purification. EMCV RNA was produced as previously described ${ }^{6}$. Briefly, Vero cells were infected with EMCV $(\mathrm{MOI}=0.1)$ for $16 \mathrm{~h}$, and total RNA was isolated using the Direct-zol RNA extraction kit (Zymo Research) as per the manufacturer's instructions. Mock RNA and SCoV2 RNA were produced by isolating total RNA from uninfected or SCoV2-infected (MOI $=1$ for $24 \mathrm{~h}$ ) Vero-hACE2 cells. RABV $\mathrm{L}_{\mathrm{Le}}$ was generated by in vitro transcription using the MEGAshortscript T7 Transcription Kit (Invitrogen) as previously described ${ }^{53}$.

Virus infection and titration. All viral infections were performed by inoculating cells with the virus inoculum diluted in MEM or DMEM containing 2\% FBS. After 1-2h, the virus inoculum was removed and replaced with the complete growth medium (MEM or DMEM containing 10\% FBS) and cells were further incubated for the indicated times. EMCV titration was performed either on Vero cells using the median tissue culture infectious dose $\left(\mathrm{TCID}_{50}\right)$ methodology as previously described ${ }^{54}$, or on BHK-21 cells by plaque assay. The titres of ZIKV were determined by plaque assay on Vero cells as previously described ${ }^{47}$. Titration of $\mathrm{SCoV} 2$ was performed on Vero-hACE2 cells by plaque assay.

Flow cytometry. To quantify the percentage of DENV-infected cells, reconstituted MDA5 KO HEK293 cells were washed with phosphate-buffered saline (PBS; Gibco) and fixed with 4\% (v:v) formaldehyde in PBS at room temperature for
30 min. Cells were subsequently permeabilized with $1 \times$ BD Perm/Wash buffer (BD Biosciences) for $15 \mathrm{~min}$ and incubated with an anti-flavivirus $\mathrm{E}$ antibody (4G2; 1:100 in $1 \times \mathrm{BD}$ Perm/Wash buffer) at $4^{\circ} \mathrm{C}$ for $30 \mathrm{~min}$. Cells were further washed three times with $1 \times$ BD Perm/Wash buffer and incubated with a goat anti-mouse Alexa Fluor-488-conjugated secondary antibody (1:500 in 1× BD Perm/Wash buffer; Invitrogen, catalogue no. A10667) at $4{ }^{\circ} \mathrm{C}$ for $30 \mathrm{~min}$ in the dark. After washing three times with $1 \times$ BD Perm/Wash buffer, cells were analysed on a FACSCalibur flow cytometer (BD Biosciences). Data analysis was performed using the FlowJo software.

Virus protection assay. The culture supernatants from mutant or WT EMCV-infected NHLFs or RIG-I KO HEK293 cells were UV inactivated in a biosafety cabinet under a UV-C lamp $(30 \mathrm{~W})$ at a dose of $5,000 \mu \mathrm{J} \mathrm{cm}^{-2}$ for $15 \mathrm{~min}$. Complete inactivation of EMCV was confirmed by plaque assay on BHK-21 cells. The inactivated supernatants were then transferred on to fresh Vero cells for $24 \mathrm{~h}$, and the primed Vero cells were subsequently infected with ZIKV $(\mathrm{MOI}=0.002-2)$ for $72 \mathrm{~h}$, or with EMCV (MOI $=0.001-0.1)$ for $40 \mathrm{~h}$. ZIKV-positive cells were determined by immunostaining with anti-flavivirus E antibody (4G2) and visualized using the KPL TrueBlue peroxidase substrate (SeraCare). EMCV-induced cytopathic effect was visualized by Coomassie Blue staining.

Statistical analysis. The two-tailed, unpaired Student's $t$-test was used to compare differences between two experimental groups in all cases. Significant differences are denoted by ${ }^{\star} P<0.05,{ }^{* *} P<0.01$ or ${ }^{\star * *} P<0.001$. Pre-specified effect sizes were not assumed and, in general, three biological replicates $(n)$ for each condition were used.

Reporting Summary. Further information on research design is available in the Nature Research Reporting Summary linked to this article.

\section{Data availability}

The data that support the findings of the present study are available from the corresponding author upon request. Source data are provided with this paper.

Received: 16 April 2020; Accepted: 24 February 2021; Published online: 16 March 2021

\section{References}

1. Liu, G. \& Gack, M. U. Distinct and orchestrated functions of RNA sensors in innate. Immunity 53, 26-42 (2020).

2. $\mathrm{Wu}, \mathrm{J}$. \& Chen, Z. J. Innate immune sensing and signaling of cytosolic nucleic acids. Annu. Rev. Immunol. 32, 461-488 (2014).

3. Chow, K. T., Gale, M.Jr \& Loo, Y. M. RIG-I and other RNA sensors in antiviral immunity. Annu. Rev. Immunol. 36, 667-694 (2018).

4. Schlee, M. Master sensors of pathogenic RNA-RIG-I like receptors. Immunobiology 218, 1322-1335 (2013).

5. Rehwinkel, J. \& Gack, M. U. RIG-I-like receptors: their regulation and roles in RNA sensing. Nat. Rev. Immunol. 20, 537-551 (2020).

6. Wies, E. et al. Dephosphorylation of the RNA sensors RIG-I and MDA5 by the phosphatase PP1 is essential for innate immune signaling. Immunity 38, 437-449 (2013)

7. Gack, M. U. et al. TRIM25 RING-finger E3 ubiquitin ligase is essential for RIG-I-mediated antiviral activity. Nature 446, 916-920 (2007).

8. Chiang, C. \& Gack, M. U. Post-translational control of intracellular pathogen sensing pathways. Trends Immunol. 38, 39-52 (2017).

9. Lazear, H. M., Schoggins, J. W. \& Diamond, M. S. Shared and distinct functions of type I and type III interferons. Immunity 50, 907-923 (2019).

10. Schoggins, J. W. Interferon-stimulated genes: what do they all do? Annu. Rev. Virol. 6, 567-584 (2019).

11. Zhang, D. \& Zhang, D. E. Interferon-stimulated gene 15 and the protein ISGylation system. J. Interferon Cytokine Res. 31, 119-130 (2011).

12. Perng, Y. C. \& Lenschow, D. J. ISG15 in antiviral immunity and beyond. Nat. Rev. Microbiol. 16, 423-439 (2018).

13. Hadjadj, J. et al. Impaired type I interferon activity and inflammatory responses in severe COVID-19 patients. Science 369, 718-724 (2020).

14. Klemm, T. et al. Mechanism and inhibition of the papain-like protease, PLpro, of SARS-CoV-2. EMBO J. 39, e106275 (2020).

15. Shin, D. et al. Papain-like protease regulates SARS-CoV-2 viral spread and innate immunity. Nature 587, 657-662 (2020).

16. Kim, M. J., Hwang, S. Y., Imaizumi, T. \& Yoo, J. Y. Negative feedback regulation of RIG-I-mediated antiviral signaling by interferon-induced ISG15 conjugation. J. Virol. 82, 1474-1483 (2008)

17. Du, Y. et al. LRRC25 inhibits type I IFN signaling by targeting ISG15-associated RIG-I for autophagic degradation. EMBO J. 37, 351-366 (2018)

18. Hato, S. V. et al. The mengovirus leader protein blocks interferon-alpha/beta gene transcription and inhibits activation of interferon regulatory factor 3. Cell Microbiol. 9, 2921-2930 (2007). 
19. Deddouche, S. et al. Identification of an LGP2-associated MDA5 agonist in picornavirus-infected cells. eLife 3, e01535 (2014).

20. Durfee, L. A., Lyon, N., Seo, K. \& Huibregtse, J. M. The ISG15 conjugation system broadly targets newly synthesized proteins: implications for the antiviral function of ISG15. Mol. Cell 38, 722-732 (2010)

21. Lenschow, D. J. et al. Identification of interferon-stimulated gene 15 as an antiviral molecule during Sindbis virus infection in vivo. J. Virol. 79, 13974-13983 (2005)

22. Gack, M. U., Nistal-Villan, E., Inn, K. S., Garcia-Sastre, A. \& Jung, J. U. Phosphorylation-mediated negative regulation of RIG-I antiviral activity. J. Virol. 84, 3220-3229 (2010).

23. Nistal-Villan, E. et al. Negative role of RIG-I serine 8 phosphorylation in the regulation of interferon-beta production. J. Biol. Chem. 285, 20252-20261 (2010)

24. Maharaj, N. P., Wies, E., Stoll, A. \& Gack, M. U. Conventional protein kinase C-alpha (PKC-alpha) and PKC-beta negatively regulate RIG-I antiviral signal transduction. J. Virol. 86, 1358-1371 (2012).

25. Davis, M. E. et al. Antagonism of the phosphatase PP1 by the measles virus $\mathrm{V}$ protein is required for innate immune escape of MDA5. Cell Host Microbe 16, 19-30 (2014).

26. Lin, J. P., Fan, Y. K. \& Liu, H. M. The 14-3-3eta chaperone protein promotes antiviral innate immunity via facilitating MDA5 oligomerization and intracellular redistribution. PLoS Pathog. 15, e1007582 (2019).

27. Wu, B. et al. Structural basis for dsRNA recognition, filament formation, and antiviral signal activation by MDA5. Cell 152, 276-289 (2013).

28. Yu, Q., Qu, K. \& Modis, Y. Cryo-EM structures of MDA5-dsRNA filaments at different stages of ATP hydrolysis. Mol. Cell 72 999-1012 (2018).

29. Bruns, A. M., Leser, G. P., Lamb, R. A. \& Horvath, C. M. The innate immune sensor LGP2 activates antiviral signaling by regulating MDA5-RNA interaction and filament assembly. Mol. Cell 55, 771-781 (2014).

30. Uchikawa, E. et al. Structural analysis of dsRNA binding to anti-viral pattern recognition receptors LGP2 and MDA5. Mol. Cell 62, 586-602 (2016).

31. Malakhova, O. A. et al. UBP43 is a novel regulator of interferon signaling independent of its ISG15 isopeptidase activity. EMBO J. 25, 2358-2367 (2006).

32. Zhang, X. et al. Human intracellular ISG15 prevents interferon-alpha/beta over-amplification and auto-inflammation. Nature 517, 89-93 (2015).

33. Harcourt, B. H. et al. Identification of severe acute respiratory syndrome coronavirus replicase products and characterization of papain-like protease activity. J. Virol. 78, 13600-13612 (2004).

34. Roth-Cross, J. K., Bender, S. J. \& Weiss, S. R. Murine coronavirus mouse hepatitis virus is recognized by MDA5 and induces type I interferon in brain macrophages/microglia. J. Virol. 82, 9829-9838 (2008).

35. Menachery, V. D. et al. Attenuation and restoration of severe acute respiratory syndrome coronavirus mutant lacking 2 '-O-methyltransferase activity. J. Virol 88, 4251-4264 (2014).

36. Bekes, M. et al. Recognition of Lys48-linked di-ubiquitin and deubiquitinating activities of the SARS coronavirus papain-like protease. Mol. Cell 62, 572-585 (2016)

37. Childs, K. et al. mda-5, but not RIG-I, is a common target for paramyxovirus V proteins. Virology 359, 190-200 (2007).

38. Shi, H. X. et al. Positive regulation of interferon regulatory factor 3 activation by Herc5 via ISG15 modification. Mol. Cell Biol. 30, 2424-2436 (2010).

39. Blanco-Melo, D. et al. Imbalanced host response to SARS-CoV-2 drives development of COVID-19. Cell 181, 1036-1045 (2020).

40. Speer, S. D. et al. ISG15 deficiency and increased viral resistance in humans but not mice. Nat. Commun. 7, 11496 (2016).

41. Errett, J. S., Suthar, M. S., McMillan, A., Diamond, M. S. \& Gale, M. Jr. The essential, nonredundant roles of RIG-I and MDA5 in detecting and controlling West Nile virus infection. J. Virol. 87, 11416-11425 (2013).

42. Wolff, G. et al. A molecular pore spans the double membrane of the coronavirus replication organelle. Science 369, 1395-1398 (2020).

43. Major, E. O. et al. Establishment of a line of human fetal glial cells that supports JC virus multiplication. Proc. Natl Acad. Sci. USA 82 1257-1261 (1985).
44. Hertzog, J. et al. Infection with a Brazilian isolate of Zika virus generates RIG-I stimulatory RNA and the viral NS5 protein blocks type I IFN induction and signaling. Eur. J. Immunol. 48, 1120-1136 (2018).

45. Cerikan, B. et al. Cell-intrinsic adaptation arising from chronic ablation of a key rho GTPase regulator. Dev. Cell 39, 28-43 (2016).

46. Chan, Y. K. \& Gack, M. U. A phosphomimetic-based mechanism of dengue virus to antagonize innate immunity. Nat. Immunol. 17, 523-530 (2016).

47. Riedl, W. et al. Zika virus NS3 mimics a cellular 14-3-3-binding motif to antagonize RIG-I- and MDA5-mediated innate immunity. Cell Host Microbe 26, 493-503 (2019).

48. Ulane, C. M., Rodriguez, J. J., Parisien, J. P. \& Horvath, C. M. STAT3 ubiquitylation and degradation by mumps virus suppress cytokine and oncogene signaling. J. Virol. 77, 6385-6393 (2003).

49. Oudshoorn, D. et al. HERC6 is the main E3 ligase for global ISG15 conjugation in mouse cells. PLoS ONE 7, e29870 (2012).

50. Kim, D. K. et al. A comprehensive, flexible collection of SARS-CoV-2 coding regions. G3 (Bethesda) 10, 3399-3402 (2020).

51. Chiang, C. et al. The human papillomavirus E6 oncoprotein targets USP15 and TRIM25 to suppress RIG-I-mediated innate immune signaling. J. Virol. 92, e01737-17 (2018).

52. Gack, M. U. et al. Roles of RIG-I N-terminal tandem CARD and splice variant in TRIM25-mediated antiviral signal transduction. Proc. Natl Acad. Sci. USA 105, 16743-16748 (2008).

53. Chiang, J. J. et al. Viral unmasking of cellular 5S rRNA pseudogene transcripts induces RIG-I-mediated immunity. Nat. Immunol. 19 53-62 (2018).

54. Sparrer, K. M. J. et al. TRIM23 mediates virus-induced autophagy via activation of TBK1. Nat. Microbiol. 2, 1543-1557 (2017).

\section{Acknowledgements}

We thank D. Lenschow (Washington University in St. Louis), E. Schiebel (University of Heidelberg), E. Cahir-McFarland (Biogen), J. Rehwinkel (University of Oxford), F. J. M. van Kuppeveld (Utrecht University), K.-K. Conzelmann (LMU, Munich), S. Goodbourn (University of London), S. C. Baker (Loyola University Chicago), B. R. tenOever (Icahn School of Medicine at Mount Sinai), A. García-Sastre (Icahn School of Medicine at Mount Sinai) and J. U. Jung (Cleveland Clinic Lerner Research Institute) for providing reagents. We also thank S. Tavakoli and J. Poole for their help in the BSL-3 facility at the Cleveland Clinic Florida Research and Innovation Center. The present study was supported in part by the US National Institutes of Health (grant nos. R01 AI087846 and R01 AI127774 to M.U.G.)

\section{Author contributions}

G.L., J.-H.L., Z.M.P. and M.U.G. designed the experiments. G.L., J.-H.L., Z.M.P., D.A., M.v.G., W.R., J.J.C., M.E.D.-G., E.W. and C.C. performed the experiments. G.L., J.-H.L., Z.M.P., D.A., M.v.G., W.R. and M.U.G. analysed data. M.U.G conceived the study. G.L., J.-H.L., Z.M.P. and M.U.G. wrote the manuscript, with input from all the authors.

\section{Competing interests}

The authors declare no competing interests.

\section{Additional information}

Extended data is available for this paper at https://doi.org/10.1038/s41564-021-00884-1. Supplementary information The online version contains supplementary material available at https://doi.org/10.1038/s41564-021-00884-1.

Correspondence and requests for materials should be addressed to M.U.G.

Peer review information Nature Microbiology thanks Benjamin Hale, Ana Fernandez-Sesma, Nicolas Vabret and the other, anonymous, reviewer(s) for their contribution to the peer review of this work.

Reprints and permissions information is available at www.nature.com/reprints.

Publisher's note Springer Nature remains neutral with regard to jurisdictional claims in published maps and institutional affiliations.

(C) The Author(s), under exclusive licence to Springer Nature Limited 2021 

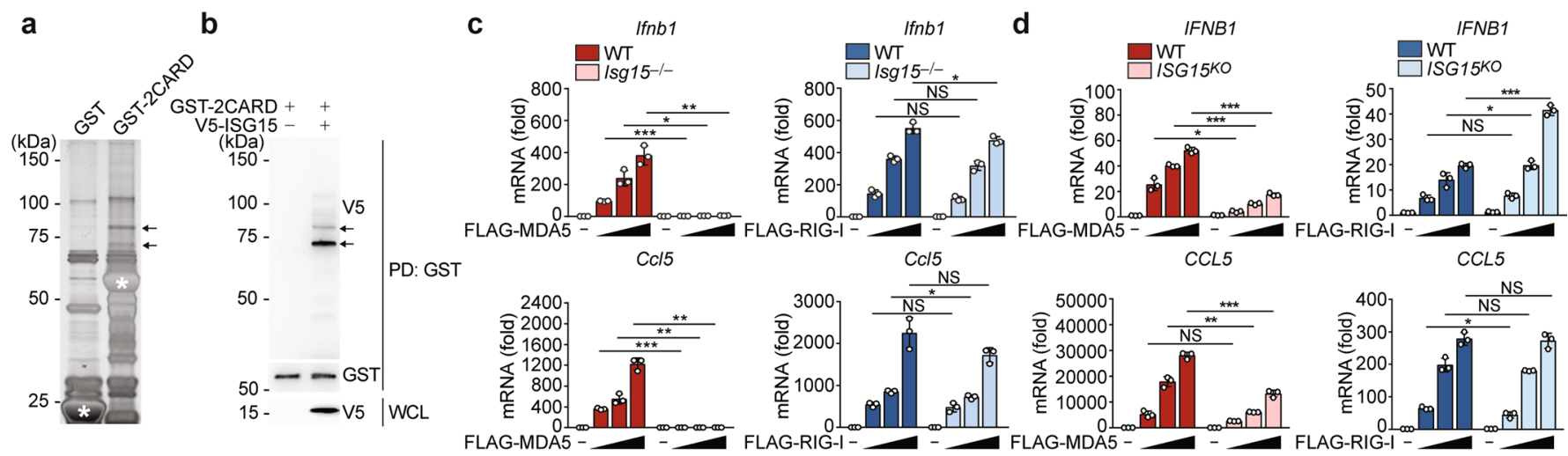

e

f

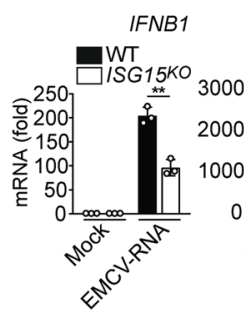

g
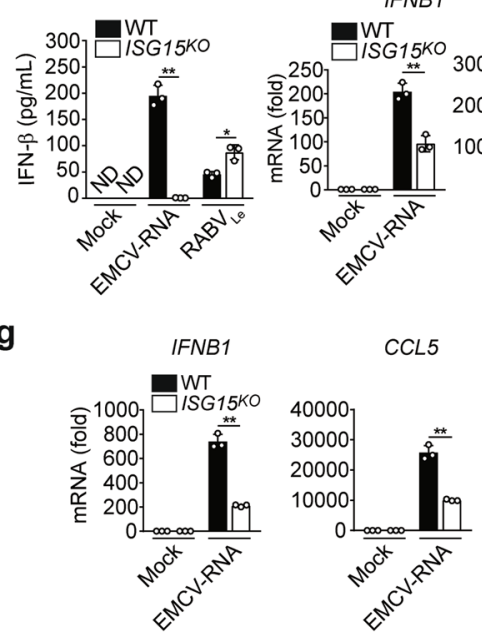

CCL5

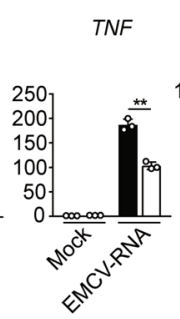

MDA5 h

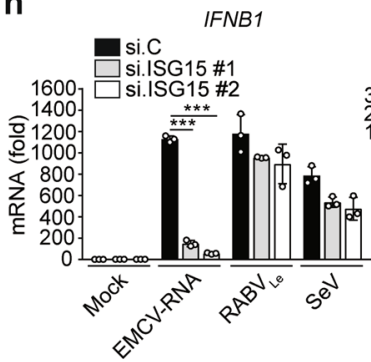

i

ISG15

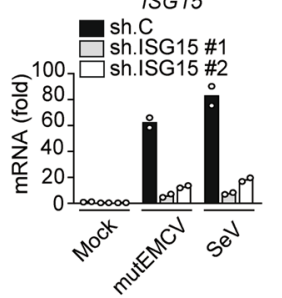

CCL5

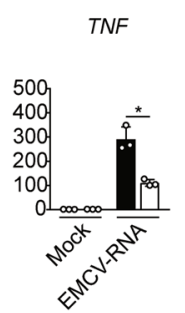

MDA5

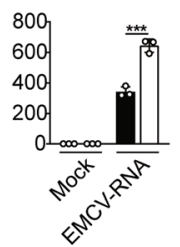

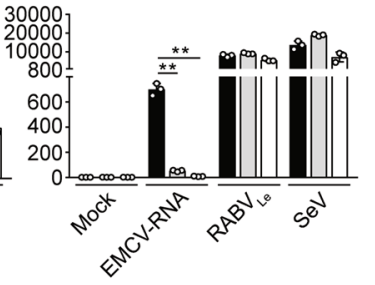

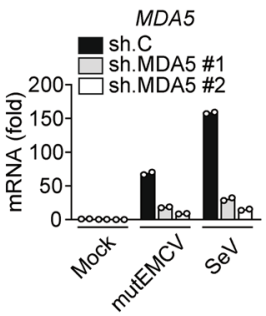

Extended Data Fig. 1 | ISG15 is required for MDA5, but not RIG-I, mediated signal transduction. a, Silver-stained affinity-purified GST and GST-MDA52CARD from transiently transfected HEK293T cells. Asterisks denote the GST and GST-MDA5-2CARD (aa 1-295) proteins. Arrows indicate the bands that identified ISG15 by MS analysis. b, GST-MDA5-2CARD ISGylation in transiently transfected HEK293T cells with or without co-expressed V5-ISG15, determined by GST pulldown (PD) and immunoblot (IB) with anti-V5 and anti-GST. Whole cell lysates (WCLs) were probed by IB with anti-V5. c, d, RT-qPCR analysis of IFNB1 and CCL5 transcripts in WT and Isg15 ${ }^{-/-}$MEFs (c) or WT and ISG15 KO HeLa cells (d) that were transfected with empty vector or increasing amounts of FLAG-MDA5 or FLAG-RIG-I for 40 h. e, ELISA of IFN- $\beta$ in the supernatants of WT or ISG15 KO HeLa cells that were

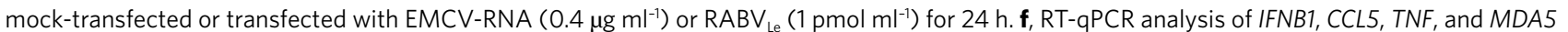

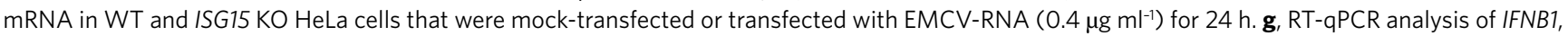
CCL5, TNF, and MDA5 mRNA in WT and ISG15 KO HAP-1 cells that were stimulated as in (f). h, RT-qPCR analysis of IFNB1 and CCL5 mRNA in NHLFs that were transfected with the indicated siRNAs for $30 \mathrm{~h}$ and then mock-stimulated or transfected with EMCV-RNA $\left(0.4 \mu \mathrm{g} \mathrm{ml} \mathrm{l}^{-1}\right)$ or RABV $\mathrm{Le}\left(1 \mathrm{pmol} \mathrm{ml}^{-1}\right)$, or infected with SeV $\left(10 \mathrm{HAU} \mathrm{ml}^{-1}\right)$ for $16 \mathrm{~h}$. i, RT-qPCR analysis of ISG15 and MDA5 mRNA in PBMCs that were transduced for $40 \mathrm{~h}$ with the indicated shRNA lentiviral particles and then infected with mutEMCV $(\mathrm{MOI}=10)$ or $\mathrm{SeV}\left(200 \mathrm{HAU} \mathrm{ml} \mathrm{H}^{-1}\right)$ for $8 \mathrm{~h}$. Data represent at least two independent experiments with similar results (mean \pm s.d. of $n=3$ biological replicates in $\mathbf{c}-\mathbf{h}$; mean of $n=2$ biological replicates in $\mathbf{i}$ ). ${ }^{\star} P<0.05,{ }^{\star \star} P<0.01,{ }^{\star \star \star} P<0.001$ (two-tailed, unpaired Student's t-test). NS, not significant; ND, not detected. 
a

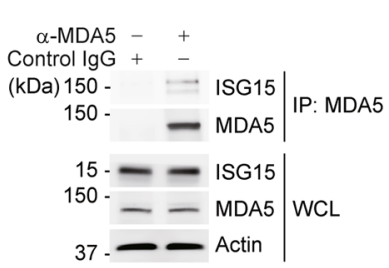

C

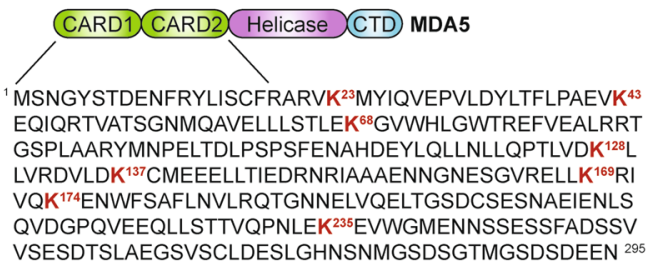

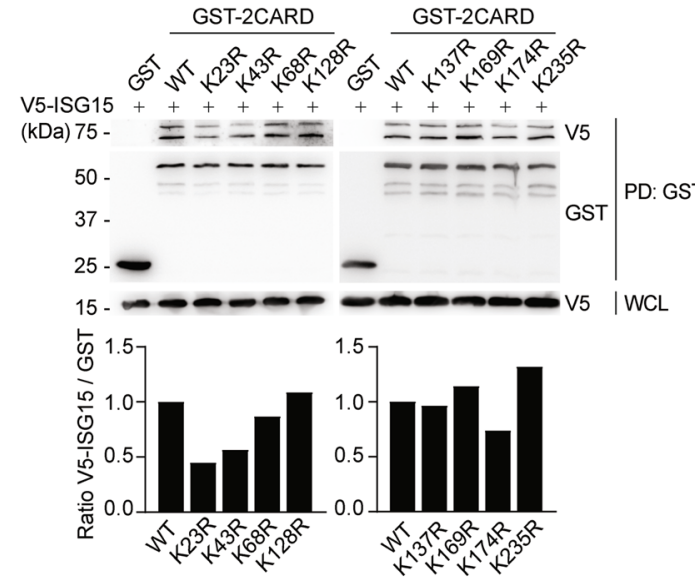

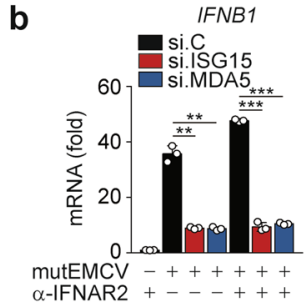

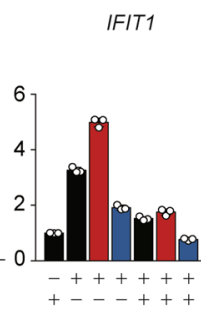

RSAD2

ISG15

MDA5

d

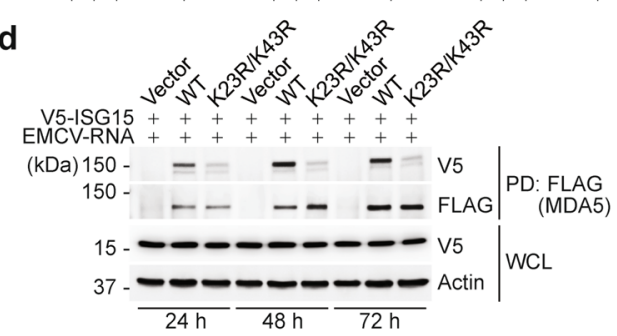

f

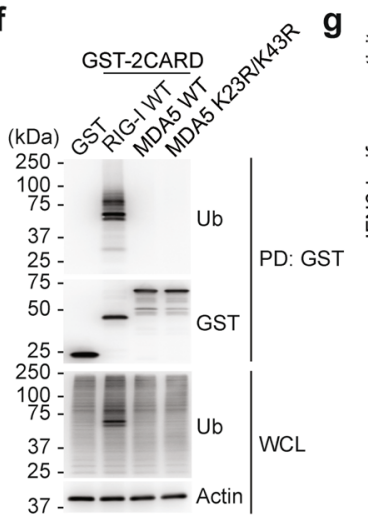

i

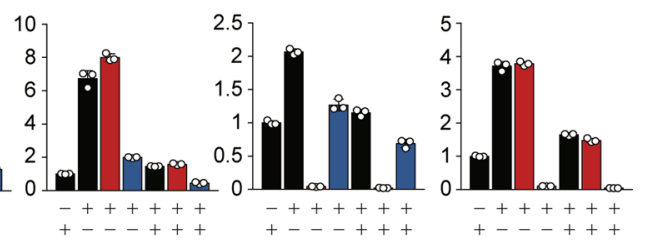

e<smiles>[14CH]=[14CH][GeH3]</smiles>

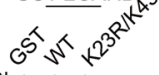
Myc-UBE2I + + + FLAG-SUMO1 +

$$
\begin{aligned}
& \text { (kDa) } 50-\angle \equiv=\text { GST |PD: FLAG }
\end{aligned}
$$

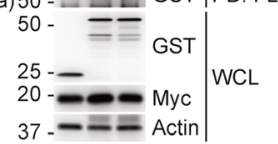

h
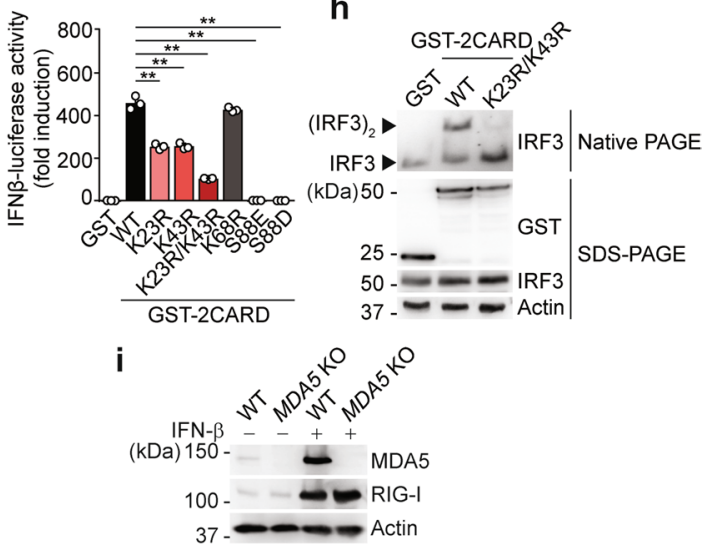

Extended Data Fig. 2 | ISGylation at K23 and K43 is essential for MDA5 activation. a, ISGylation of endogenous MDA5 in uninfected NHLFs, determined by IP with anti-MDA5 (or an IgG isotype control) and IB with anti-ISG15. b, RT-qPCR analysis of the indicated transcripts in NHLFs that were transfected for $30 \mathrm{~h}$ with the indicated siRNAs and then infected with mutEMCV (MOI 0.005) for $16 \mathrm{~h}$ in the absence or presence of anti-IFNAR2 (2 $\mu \mathrm{g}$ $\mathrm{ml}^{-1}$ ). c, Upper panels: Amino acid sequence of the MDA5 CARDs. Lysine (K) residues are highlighted in red. CTD, C-terminal domain. Middle panels: ISGylation of GST-MDA5-2CARD WT or mutants in HEK293T cells that co-expressed V5-ISG15, determined by GST PD and IB with anti-V5. Lower panels: Densitometric analysis of the ISGylation levels of GST-MDA5-2CARD WT and mutants, normalized to protein levels in GST PD. d, ISGylation of FLAG-MDA5 WT and K23R/K43R in transfected MDA5 KO HEK293 cells that co-expressed V5-ISG15 and were stimulated with EMCV-RNA (0.4 $\mu$ g $\mathrm{ml}^{-1}$ ) for the indicated times, determined by FLAG PD and IB with anti-V5. e, SUMOylation of GST-MDA5-2CARD WT and K23R/K43R in transfected HEK293T cells that co-expressed Myc-UBE2I and FLAG-SUMO1, determined by FLAG PD and IB with anti-GST. f, Ubiquitination of GST-RIG-I-2CARD WT, or GST-MDA5-2CARD WT and K23R/K43R in HEK293T cells, determined by GST PD and IB with anti-Ub. $\mathbf{g}$, IFN- $\beta$-luciferase activity in HEK293T cells transfected for $40 \mathrm{~h}$ with GST, or GST-MDA5-2CARD (GST-2CARD) WT or mutants. h, Endogenous IRF3 dimerization in HEK293T cells transfected with GST, or GST-MDA5-2CARD WT or K23R/K43R for $24 \mathrm{~h}$, determined by NativePAGE and IB with anti-IRF3. WCLs were analyzed by SDS-PAGE and IB with anti-GST, anti-IRF3, and anti-actin. i, Validation of MDA5 gene editing in SVGAs. MDA5 protein abundance in WT control and MDA5 KO SVGAs treated with IFN- $\beta\left(1,000 \mathrm{U} \mathrm{ml}^{-1}\right)$ for $16 \mathrm{~h}$, or left untreated, assessed by IB with anti-MDA5. Immunoblotting for RIG-I and actin served as control. Data represent at least two independent experiments with similar results (mean \pm s.d. of $n=3$ biological replicates in $\mathbf{b}$ and $\mathbf{g}$ ). ${ }^{\star \star} P<0.01,{ }^{\star \star \star} P<0.001$ (two-tailed, unpaired Student's t-test). 
a

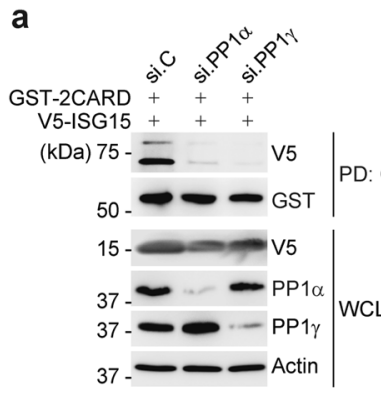

f

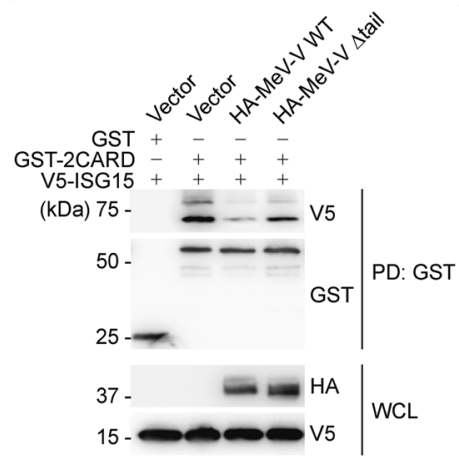

b

C
GST-2CARD

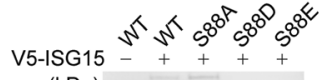

(KDa)
$75-$

50-ーயー-GST PD: GST

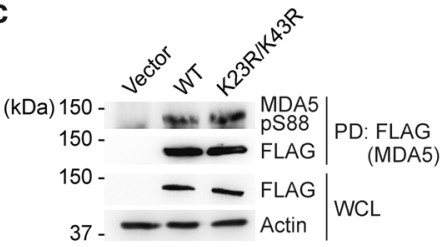

g

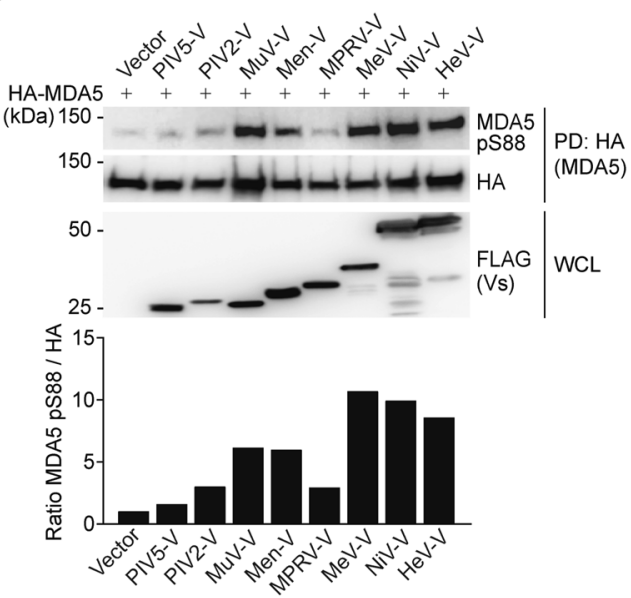

e

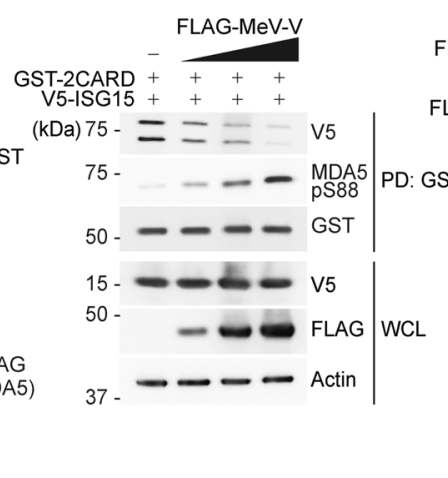

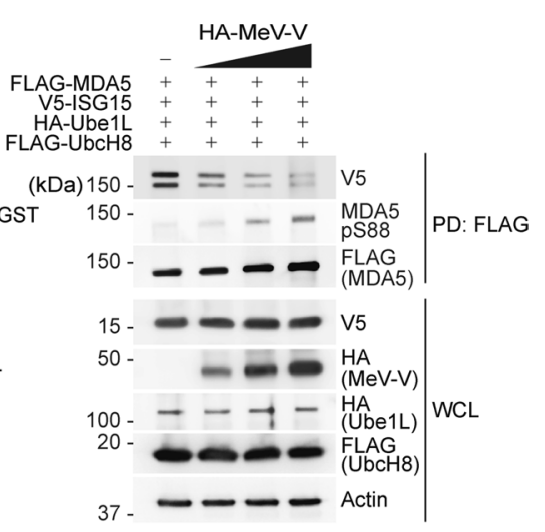

h

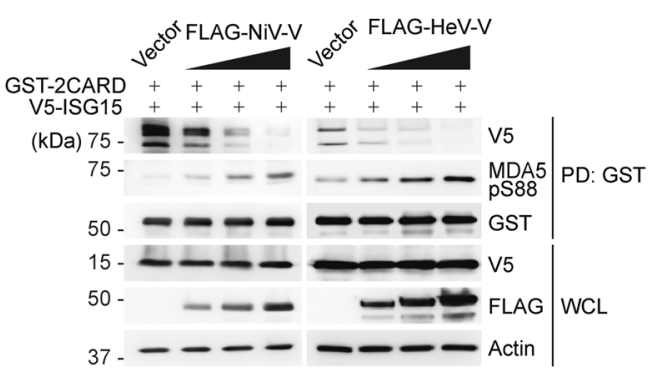

Extended Data Fig. 3 | Dephosphorylation of MDA5 induces ISGylation. a, ISGylation of GST-MDA5-2CARD in HEK293T cells transfected with V5-ISG15 and the indicated siRNAs for $48 \mathrm{~h}$, assessed by GST PD and IB with anti-V 5 and anti-GST. WCLs were probed by IB with anti-V5, anti-PP1 $\alpha$, anti-PP1 $\gamma$, and anti-actin. b. ISGylation of GST-MDA5-2CARD WT or S88A, S88D and S88E mutants in transiently transfected HEK293T cells that also co-expressed V5-ISG15, determined by GST PD and IB with anti-V5 and anti-GST forty hours after transfection. c, Phosphorylation of FLAG-MDA5 WT or K23R/K43R mutant in HEK293T cells, determined by FLAG PD and IB with anti-pS88-MDA5 and anti-FLAG. WCLs were probed by IB with anti-FLAG and anti-actin. d, ISGylation and phosphorylation of GST-MDA5-2CARD in HEK293T cells transfected with V5-ISG15 and either empty vector or increasing amounts of FLAG-MeV-V for $24 \mathrm{~h}$, determined by GST PD and IB with anti-pS88-MDA5, anti-V5, and anti-GST. e, ISGylation and phosphorylation of FLAG-tagged MDA5 in transfected HEK293T cells that co-expressed V5-ISG15, HA-Ube1L, and FLAG-UbcH8 as well as either empty vector or increasing amounts of HA-MeV-V for $24 \mathrm{~h}$, determined by FLAG PD and IB with anti-pS88-MDA5, anti-V5, and anti-FLAG. WCLs were probed by IB with the indicated antibodies. f, ISGylation of GST-MDA5-2CARD, or GST (negative control), in HEK293T cells transiently transfected with V5-ISG15 and either empty vector or HA-tagged MeV-V WT or $\triangle$ tail for $48 \mathrm{~h}$, determined by GST PD and IB with anti-V5 and anti-GST. g, Upper panel: Phosphorylation of HA-MDA5 in transfected HEK293T cells that co-expressed empty vector or the indicated FLAG-tagged paramyxoviral $V$ proteins, assessed by HA PD and IB with anti-pS88-MDA5 and anti-HA. WCLs were probed by IB with anti-FLAG. Lower panel: Densitometric analysis of the levels of MDA5 S88 phosphorylation, normalized to protein levels in HA PD. h, ISGylation and phosphorylation of GST-MDA5-2CARD in transfected HEK293T cells that co-expressed V5-ISG15 and either empty vector or increasing amounts of FLAG-NiV-V or FLAG-HeV-V for $24 \mathrm{~h}$, determined by GST PD and IB with anti-V5, anti-pS88-MDA5, and anti-GST. WCLs were probed by IB with anti-FLAG, anti-V5, and anti-actin. Data represent at least two independent experiments with similar results. 
a

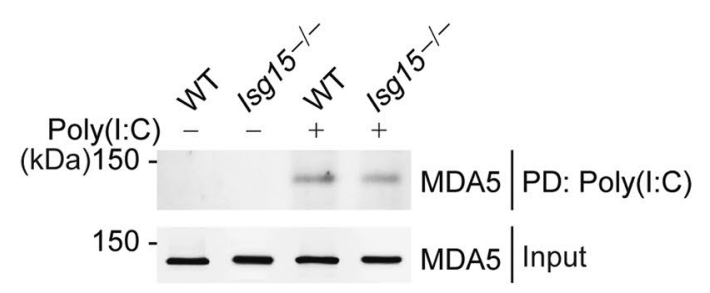

C

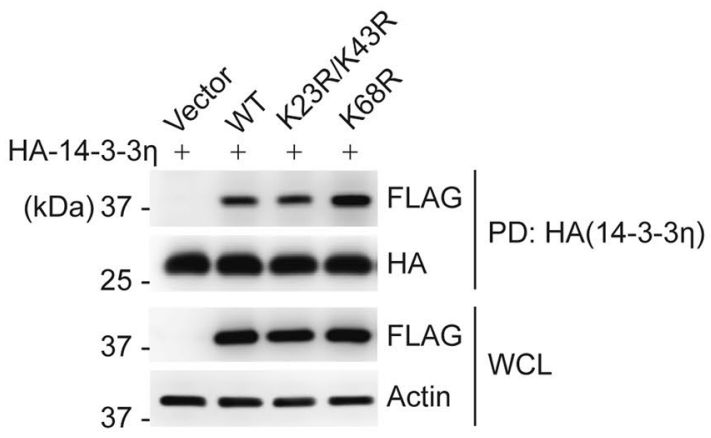

b

Poly(I:C)

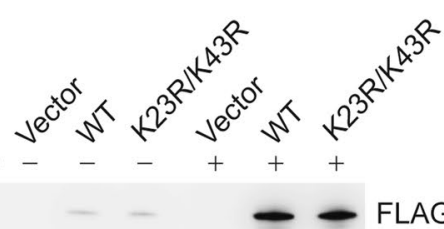

FLAG |PD: Poly(I:C)

150 - $\quad==$ FLAG $\mid$ Input

d

Extended Data Fig. 4 | CARD ISGylation does not affect the ability of MDA5 to bind RNA, 14-3-3y, or LGP2. a, In vitro RNA-binding ability of endogenous MDA5 from WT or Isg15-/- MEFs that were stimulated with IFN- $\beta\left(1,000 \mathrm{U} \mathrm{ml}^{-1}\right)$ for $24 \mathrm{~h}$, assessed by biotin-HMW-poly (I:C) PD and IB with anti-MDA5. Equal input MDA5 protein amounts were confirmed by IB with anti-MDA5. b, In vitro RNA-binding ability of FLAG-MDA5 WT and K23R/K43R from transiently transfected HEK293T cells, assessed by biotin-HMW-poly(I:C) PD and IB with anti-FLAG. Equal input FLAG-MDA5 protein amounts were

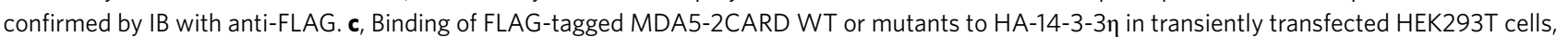
determined by HA PD and IB with anti-FLAG and anti-HA. WCLs were probed by IB with anti-FLAG and anti-actin. d, Binding of FLAG-tagged MDA5 WT and K23R/K43R to HA-tagged LGP2 in transiently transfected HEK293T cells, determined by HA PD and IB with anti-MDA5 and anti-HA. WCLs were probed by IB with anti-MDA5. Data represent at least two independent experiments with similar results. 
a

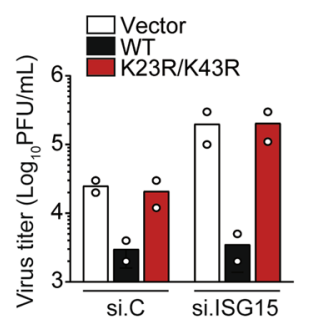

b

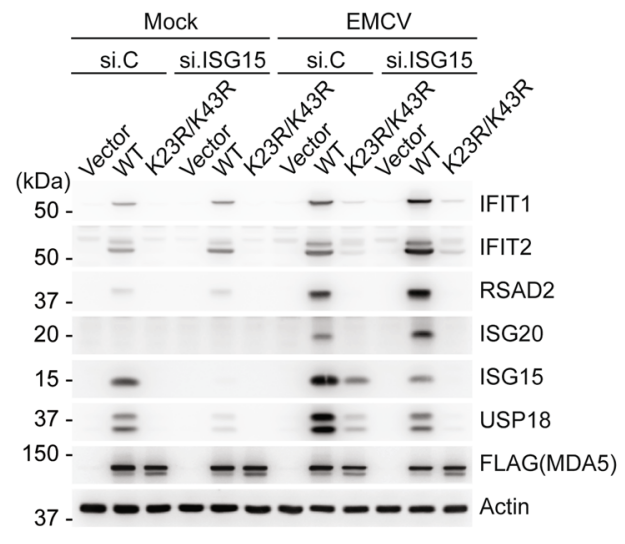

d

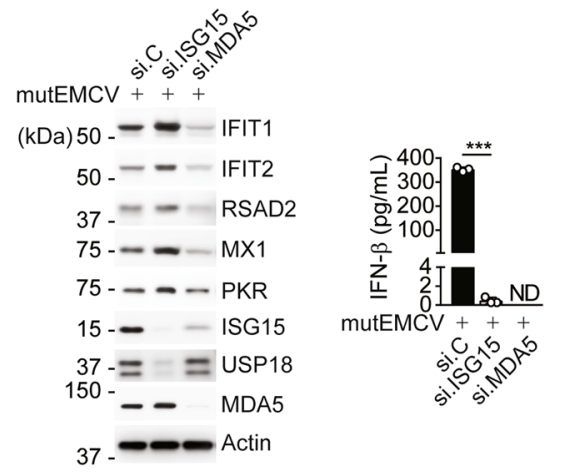

C

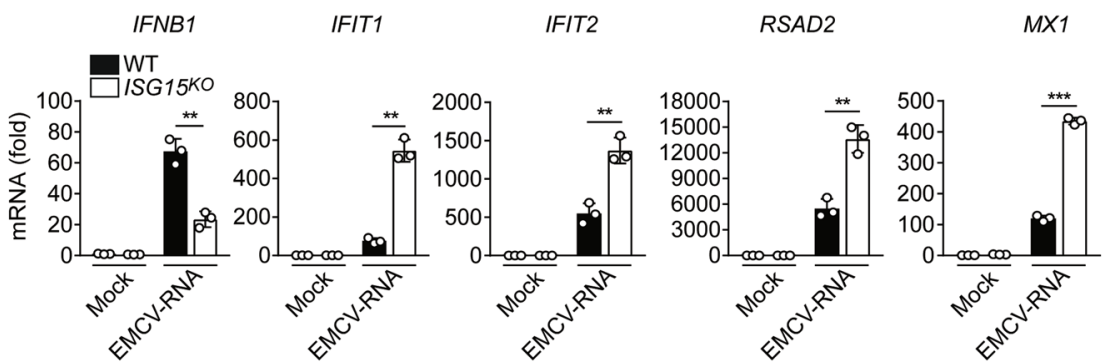

Extended Data Fig. 5 | Aberrant ISG upregulation in ISG15-deficient cells upon MDA5 stimulation. a, EMCV titers in the supernatant of RIG-I KO HEK293 cells that were transfected for $24 \mathrm{~h}$ with nontargeting control siRNA (si.C) or ISG15-specific siRNA (si.ISG15) and then transfected with either empty vector or FLAG-tagged MDA5 WT or K23R/K43R for $24 \mathrm{~h}$ prior to infection with EMCV $(\mathrm{MOI}=0.001)$ for $16 \mathrm{~h}$, determined by plaque assay. b, Protein abundance of the indicated ISGs and USP18 in mock-infected or EMCV-infected (MOI=0.001 for $16 \mathrm{~h}$ ) RIG-I KO HEK293 cells that were transfected with the indicated siRNAs, and $24 \mathrm{~h}$ later, transfected with empty vector or FLAG-MDA5 WT or K23/K43R for $24 \mathrm{~h}$, determined by IB with the indicated antibodies. WCLs were further immunoblotted with anti-FLAG and anti-actin. $\mathbf{c}$, RT-qPCR analysis of IFNB1 and ISG transcripts in WT and ISG15 KO HeLa cells that were mock-treated or transfected with EMCV-RNA $\left(0.4 \mu \mathrm{g} \mathrm{ml}^{-1}\right)$ for $16 \mathrm{~h}$. d, Left panel: Protein abundance of the indicated ISGs and USP18 in NHLFs that were transfected for $40 \mathrm{~h}$ with the indicated siRNAs and then infected with mutEMCV $(\mathrm{MOI}=0.1)$ for $16 \mathrm{~h}$, determined by IB with the indicated antibodies. Right panel: ELISA of IFN- $\beta$ from supernatants of NHLFs from the same experiment (left panel). Data represent at least two independent experiments with similar results (mean of $n=2$ biological replicates in $\mathbf{a}$; mean \pm s.d. of $n=3$ biological replicates in $\mathbf{c}$ and $\mathbf{d}$ ). ${ }^{\star \star} p<0.01$, ${ }^{* *} p<0.001$ (two-tailed, unpaired Student's t-test). ND, not detected. 
a

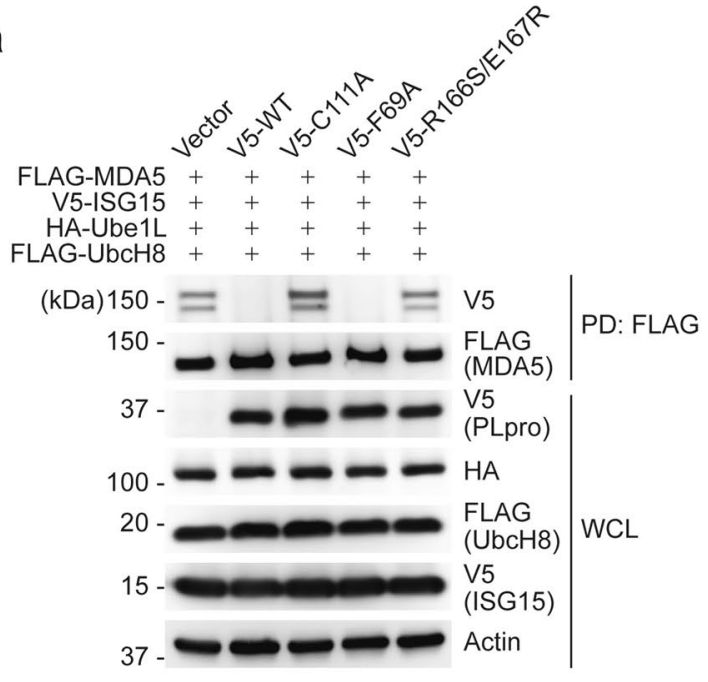

b

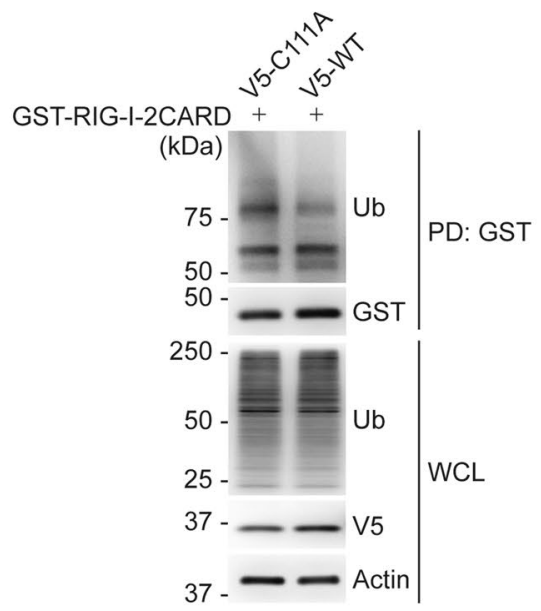

C

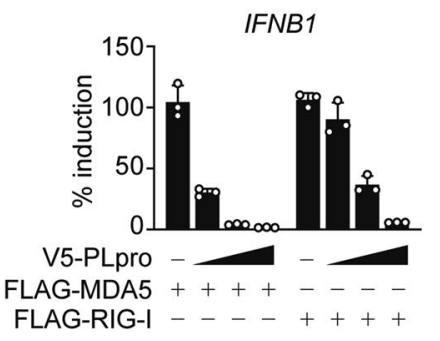

Extended Data Fig. 6 | SCoV2 PLpro does not affect RIG-I ubiquitination and preferentially antagonizes the MDA5 pathway. a, ISGylation of FLAGMDA5 in HEK293T cells that were co-transfected for $20 \mathrm{~h}$ with vector or V5-tagged SCoV2 PLpro WT or mutants, along with V5-ISG15, HA-Ube1L, and FLAG-UbcH8, determined by FLAG PD and IB with anti-V5 and anti-FLAG. WCLs were probed by IB with anti-V5, anti-HA, anti-FLAG, and anti-actin. b, Ubiquitination of GST-RIG-I-2CARD in HEK293T cells that were co-transfected with V5-tagged SCoV2 PLpro WT or C111A for 24 h, determined by GST PD and IB with anti-Ub and anti-GST. WCLs were probed by IB with anti-Ub, anti-V5, and anti-actin. c, Upper panels: RT-qPCR analysis of IFNB1 and CCL5 transcripts in HeLa cells that were transfected with FLAG-MDA5 or FLAG-RIG-I together with either empty vector or increasing amounts of V5-tagged SCoV2 PLpro (10 ng, $25 \mathrm{ng}$, and $50 \mathrm{ng}$ ) for $24 \mathrm{~h}$. Data are presented as percentage of induction relative to the values for cells transfected with the respective RLR (that is FLAG-MDA5 or FLAG-RIG-I) and vector, set to $100 \%$. Lower panels: WCLs from the same experiment were probed by IB with the indicated antibodies. Data represent at least two independent experiments with similar results (mean \pm s.d. of $n=3$ biological replicates in $\mathbf{c}$ ). 


\section{Reporting Summary}

Nature Research wishes to improve the reproducibility of the work that we publish. This form provides structure for consistency and transparency in reporting. For further information on Nature Research policies, see Authors \& Referees and the Editorial Policy Checklist.

\section{Statistics}

For all statistical analyses, confirm that the following items are present in the figure legend, table legend, main text, or Methods section.

n/a $\mid$ Confirmed

$\square$ \.he exact sample size $(n)$ for each experimental group/condition, given as a discrete number and unit of measurement

$\square$ \A statement on whether measurements were taken from distinct samples or whether the same sample was measured repeatedly

The statistical test(s) used AND whether they are one- or two-sided

$\square$ Only common tests should be described solely by name; describe more complex techniques in the Methods section.

$\bigotimes \square$ A description of all covariates tested

Х $\square$ A description of any assumptions or corrections, such as tests of normality and adjustment for multiple comparisons

A full description of the statistical parameters including central tendency (e.g. means) or other basic estimates (e.g. regression coefficient)

AND variation (e.g. standard deviation) or associated estimates of uncertainty (e.g. confidence intervals)

$\square$ For null hypothesis testing, the test statistic (e.g. $F, t, r$ ) with confidence intervals, effect sizes, degrees of freedom and $P$ value noted

$\square$ Give P values as exact values whenever suitable.

Х $\square$ For Bayesian analysis, information on the choice of priors and Markov chain Monte Carlo settings

Х $\square$ For hierarchical and complex designs, identification of the appropriate level for tests and full reporting of outcomes

\ $\square$ Estimates of effect sizes (e.g. Cohen's $d$, Pearson's $r$ ), indicating how they were calculated

Our web collection on statistics for biologists contains articles on many of the points above.

\section{Software and code}

Policy information about availability of computer code

Data collection ABI 7500 software, BD FACS DIVA Software

Data analysis GraphPad Prism 8.4.3, MS Excel, Flowjo, Image J, ABI 7500 software

For manuscripts utilizing custom algorithms or software that are central to the research but not yet described in published literature, software must be made available to editors/reviewers. We strongly encourage code deposition in a community repository (e.g. GitHub). See the Nature Research guidelines for submitting code \& software for further information.

\section{Data}

Policy information about availability of data

All manuscripts must include a data availability statement. This statement should provide the following information, where applicable:

- Accession codes, unique identifiers, or web links for publicly available datasets

- A list of figures that have associated raw data

- A description of any restrictions on data availability

There are no restrictions on availability of data. The data that support the findings of this study are available from the corresponding author upon request.

\section{Field-specific reporting}

Please select the one below that is the best fit for your research. If you are not sure, read the appropriate sections before making your selection. $\bigotimes$ Life sciences $\quad \square$ Behavioural \& social sciences $\square$ Ecological, evolutionary \& environmental sciences 


\section{Life sciences study design}

All studies must disclose on these points even when the disclosure is negative.

Sample size The number of experiments and replicates was based on standard practices. In general, two or three independent experiments were conducted with three biological replicates or technical replicates where applicable. Details for each experiment are included in the respective figure legend.

Data exclusions $n / a$

Replication The experimental findings were reliably reproduced. See details in respective figure legends.

Randomization n/a

Blinding n/a

\section{Reporting for specific materials, systems and methods}

We require information from authors about some types of materials, experimental systems and methods used in many studies. Here, indicate whether each material, system or method listed is relevant to your study. If you are not sure if a list item applies to your research, read the appropriate section before selecting a response.

Materials \& experimental systems

\begin{tabular}{l|l}
\hline n/a & Involved in the study \\
$\square$ & $\bigotimes$ Antibodies \\
$\square$ & $\square$ Eukaryotic cell lines \\
$\square$ & $\square$ Animals and other organisms \\
$\square$ Clinical data
\end{tabular}

Methods

$\mathrm{n} / \mathrm{a}$ Involved in the study

$\bigotimes \square$ ChIP-seq

$\square \bigotimes$ Flow cytometry

$\bigotimes \square$ MRI-based neuroimaging

\section{Antibodies}

Antibodies used

Details about all antibodies used in our study are described in the Methods section of the manuscript including vendor name, Cat\# and clone.

Validation

All antibodies, except the anti-phospho-MDA5 (Ser88) and anti-MDA5 antibodies, were purchased from commercial vendors who have validated the antibodies for the use of Western blot, Co-immunoprecipitation, and/or FACS analysis. The antiphospho-MDA5 (Ser88) antibody was previously validated by the Gack laboratory and has been published (Wies et al., Immunity 2013). The anti-MDA5 antibodies were provided by Dr. Jan Rehwinkel (Oxford University) and has been published (Hertzog et al., Eur J Immunol 2018).

\section{Eukaryotic cell lines}

Policy information about cell lines

Cell line source(s)

NHLF (Lonza), HEK293T (ATCC), Vero (ATCC), BHK-21 (ATCC), Aedes albopictus clone C6/36 (ATCC), HAP-1 WT and ISG15 KO cells (Horizon Discovery). Human PBMCs were isolated from unidentified healthy donor peripheral blood (HemaCare). The WT and Isg15 -/- MEF, WT and ISG15 KO HeLa, SVGA, MDA5 KO HEK293, RIG-I KO HEK293, HEK293T-hACE2, Vero-hACE2, and A549-hACE2 cells were obtained from other investigators (details are described in the Methods section of our manuscript).

Authentication

Cell lines from ATCC, Lonza, and Horizon Discovery were authenticated by the vendors and were not further authenticated in our laboratory. Cell lines that were obtained and validated by other groups were not further authenticated. All ISG15 KO or MDA5 KO cell lines were validated by confirming the absence of ISG15 or MDA5 protein expression by immunoblotting.

Mycoplasma contamination Cell lines have been regularly tested for potential mycoplasma contamination by PCR and/or using the MycoAlert Kit (Lonza).

Commonly misidentified lines (See ICLAC register) 


\section{Plots}

Confirm that:

\The axis labels state the marker and fluorochrome used (e.g. CD4-FITC).

Х The axis scales are clearly visible. Include numbers along axes only for bottom left plot of group (a 'group' is an analysis of identical markers).

\All plots are contour plots with outliers or pseudocolor plots.

$\bigotimes$ A numerical value for number of cells or percentage (with statistics) is provided.

\section{Methodology}

Sample preparation

Instrument

Software

Cell population abundance

Gating strategy

\begin{abstract}
Cells were harvested and then pelleted by centrifugation at 300 x g for 5 min. Cells were washed with PBS and fixed with $4 \%$ (v/v) formaldehyde in PBS at room temperature for $30 \mathrm{~min}$, followed by washing cells twice with PBS. For antibody staining, cells were incubated with 1× BD Perm/Wash buffer (BD Biosciences), followed by staining with primary (anti-flavivirus E [4G2] ; 1:100) and secondary (goat anti-mouse Alexa 488; 1:500) antibody at 4 degree in 1× BD Perm/Wash buffer for 30 min each.
\end{abstract}

LSR Fortessa flow cytometer (BD Biosciences)

FACS Diva (BD Biosciences), FlowJo (Tree Star)

$\mathrm{n} / \mathrm{a}$

The main cell population was gated in the FCS/SSC blot and then analyzed in the Alexa 488 channel. Positive cells were above cutoff value, which is set to $98 \%$ of measure events of the negative control.

Tick this box to confirm that a figure exemplifying the gating strategy is provided in the Supplementary Information. 